\title{
ON THE CONVERGENCE OF SPH METHOD FOR SCALAR CONSERVATION LAWS WITH BOUNDARY CONDITIONS*
}

\author{
BACHIR BEN MOUSSA ${ }^{\dagger}$
}

\begin{abstract}
This paper is the third of a series where the convergence analysis of SPH method for multidimensional conservation laws is analyzed. In this paper, two original numerical models for the treatment of boundary conditions are elaborated. To take into account nonlinear effects in agreement with Bardos, LeRoux and Nedelec boundary conditions ([1], [14]), the state at the boundary is computed by solving appropriate Riemann problems. The first numerical model is developed around the idea of boundary forces in surrounding walls, recently initiated in [33] by Monaghan in his simulation of gravity currents. The second one extends the well-known approach of ghost particles for plane boundaries to the case of general curved boundaries. The convergence analysis in $L_{l o c}^{p}(p<\infty)$ is achieved thanks to the uniqueness result of measure-valued solutions recently established in [3] for $L^{\infty}$ initial and boundary data.
\end{abstract}

Key words. SPH method, numerical modeling, boundary conditions, conservation laws, measure- valued solutions, convergence

AMS subject classifications. 35L65,65M12,65M06

1. Introduction. In this paper, we continue the analysis of the meshfree method SPH (Smoothed Particle Hydrodynamics) for scalar nonlinear conservation laws. We consider the initial boundary value problem which remains a very challenging question for developing a mathematical framework to derive fitting and efficient numerical models with their convergence analysis. In ([4], [6]), this analysis is performed for the Cauchy problem by providing some new features of the SPH scheme in connection with finite volume methods. The new hybrid formulation turned out to be welladapted for constructing conservative and weak consistent SPH schemes. Regarding the stability of the resulting scheme, we have developed an original approach making use of a robust class of upwinding schemes based on approximate Riemann solvers, instead of the well-known technique employing an artificial viscosity (see [19]) to deal with shocks. On the light of these new features, we elaborate two numerical models to treat boundary conditions without compromising the high flexibility of SPH, which is at the origin of its popularity among the so-called gridless methods.

It is worth indicating that SPH has known since the end of the 1970's (see [29], [27] for the pioneer works) a great success through some specific domains of physics as astronomy and astrophysics, where fluids are mostly contained by their self-gravity. Thereby, boundary conditions are often considered as vanishing at the infinity and do not interfere in the effective numerical simulation. By contrast, when dealing with physical problems restricted to a domain as those we encounter in industrial applications, commercial codes seem to have been mostly developed since the 1950's using finite volume or finite element approaches. This systematic use originates from the fact that these Eulerian-based methods have known an intensive mathematical study and are clearly understood. As a result, since the 1980's, these methods are increasingly facing multiple difficulties hampering the advances of large-scale numerical simulations of sophisticated problems for which they are not clearly suited. For example those with multiple phases, which require an additional complex internal

\footnotetext{
*Received June 15, 2003; accepted for publication March 27, 2006.

${ }^{\dagger}$ Department of Applied Mathematics, University of Crete, GR-714 09 Heraklion, Crete, Greece (benmou@tem.uoc.gr).
} 
mathematical modeling to represent these phases. Therefore, a large part of the overall computational effort is expended on technical details connected with mesh adaptation and grid generation. Moreover, changes in the domain geometry and/or topology are more difficult, if not impossible, to accommodate with the existing meshing techniques. All of these problems make it that, since the 1990's, the engineering community is looking on methods which do not involve any mesh at all. Rapidly, $\mathrm{SPH}$, based on its Lagrangian and mesh-free characteristics, has shown a great ability and a high potential to handle great many industrial applications for which classical methods clearly struggle. Since then, the question of developing a solid mathematical background to reinforce the SPH formalism and particularly to formulate appropriate numerical models to treat boundary conditions, became central. Regarding the treatement of boundary conditions, two approaches have been proposed to model wall and free boundaries. The first one models wall boundaries by generating along the boundary a given set of particles in order to interact with the fluid particles through the so-called boundary forces that prevent the fluid from passing through the boundary. It is first used by Monaghan [33] in his simulation of gravity currents for nearly incompressible fluid flow. The second approach employs the so-called ghost particles generated in a neighborhood of the boundary outside of the physical domain. This technique is well-known for plane boundaries when approximating specular reflection boundary conditions. From the physical point of view, the two types of particles are endowed with similar physical properties to those of the particles that represent the flow and interact with them in a way such that the necessary boundary conditions are satisfied. For both approaches, we deal here with general curved boundaries, in particular we give new treatments of polyhedral boundaries as those encountered in industrial problems. We develop a mathematical framework for deriving these numerical models by taking into account nonlinear effects and by computing the state at the boundary by solving appropriate Riemann problems. As far as the stability analysis near the boundary is concerned, we introduce a nice formulation based on the equilibrium property for uniform fields. For a qualitative description of these models in the frame of Euler equations, we refer to Vila's paper [45], whereas numerical simulations in continuum mechanics can be found in the forthcoming paper [8]. We also refer to Randles and Libersky paper [38] and to the recent book by Lui and Lui [30].

Our second contribution in this paper concerns the convergence analysis of the two numerical approaches modeling boundary conditions. This analysis is derived by using the uniqueness result of measure-valued solutions established in [3] with Szepessy for $L^{\infty}$ initial and boundary data. As showed by Diperna in [13] and by Szepessy in [42], this convergence follows by proving that the approximate solutions are uniformly bounded in $L^{\infty}$, weakly consistent with all entropy inequalities and consistent with the initial data. In regard to the weak consistency with all entropy inequalities, one needs to show in particular the weak consistency with the boundary integral term associated to Bardos, LeRoux and Nedelec boundary conditions formulated in [1]. For that purpose, we introduce a new definition of measure valued solutions in bounded domains equivalent to the one proposed by Szepessy in [41]. The two definitions differ as far as the formulation of boundary conditions is concerned. Our formulation requires less information in terms of measure-valued solutions and turns out to be well-adapted for the convergence of numerical schemes in bounded domains.

To carry out this program, we start in section 2 with a brief review of SPH method and show how to adapt Raviart's standard accuracy results in ([39], [32]) to the case of bounded domains. We end this section by formulating our two numerical models 
of boundary conditions in the framework of a given boundary data. Section 3 will be concerned with the derivation of the SPH scheme for scalar nonlinear equations by taking into account nonlinear effects at the boundary. In section 4, we state the main convergence result (Theorem 4.1) as well as the uniqueness result of measurevalued solutions (Theorem 4.4). The remainder of the paper can roughly be seen as an existence proof of measure- valued solution as weak star limit of the approximate solutions provided by the SPH scheme.

2. SPH method and boundary conditions. Let $\Omega$ be a bounded open set in $\mathbb{R}^{d}$ with a smooth boundary $\partial \Omega$ and an outward unit normal vector $n$. Consider for $u: \Omega \times \mathbb{R}_{+} \rightarrow \mathbb{R}$ the following model of scalar nonlinear conservation law

$$
\mathcal{L}_{\mathbf{a}} u+\operatorname{div} F(u, x, t)=S(u, x, t), \quad(x, t) \in \Omega \times \mathbb{R}^{+}, \quad u(x, t) \in \mathbb{R}
$$

with the Bardos, LeRoux and Nedelec ([1], [14]) boundary condition on $\partial \Omega \times \mathbb{R}_{+}$, for all $k \in \mathbb{R}$,

$$
(\operatorname{sgn}(u(\bar{x}, t)-k)-\operatorname{sgn}(b(\bar{x}, t)-k))(F(u(\bar{x}, t), \bar{x}, t)-F(k, \bar{x}, t)) \cdot n(\bar{x}) \geq 0,
$$

and the initial condition

$$
u(\cdot, 0)=u_{0}, \quad \text { on } \Omega,
$$

where $F=\left(F_{1}, \ldots, F_{d}\right): \mathbb{R} \rightarrow \mathbb{R}^{d}, \operatorname{div}_{x} F(u, x, t)=\sum_{i=1}^{d} \partial F_{i}(u(x, t), x, t) / \partial x_{i}$ and $\mathbb{R}_{+} \equiv(0, \infty)$. The transport operator $\mathcal{L}_{\mathbf{a}} u$ and its adjoint operator $-\mathcal{L}_{\mathbf{a}}^{*} u$ (needed when writing the weak form of (1)) via the usual $L^{2}$ scalar product are defined by

$$
\mathcal{L}_{\mathbf{a}} u:=\frac{\partial u}{\partial t}+\operatorname{div}(\mathbf{a}(x, t) u) . \quad \mathcal{L}_{\mathbf{a}}^{*} u=\frac{\partial u}{\partial t}+\sum_{i=1}^{d} \mathbf{a}^{i}(x, t) \frac{\partial u}{\partial x^{i}}:=\frac{d}{d t}(u)
$$

In our model equation (1), the advection field $\mathbf{a}$ is a given smooth vector, say $\mathbf{a} \in$ $L^{\infty}\left(\mathbb{R}^{+}, W^{2, \infty}(\Omega)\right)$ together with the boundary condition $\mathbf{a}(\bar{x}, t) \cdot n(\bar{x})=0$ along the boundary $\partial \Omega$. These assumptions are very important in our convergence analysis since one avoids in the limit process the difficult problem related to the behavior of a regularized non smooth physical advection field (see [46]) which may lead to a nonlocal dispersive equation. Alternatively, the model equation (1) suggests to treat the physical advection term by including it in the generic nonlinear flux term $F(u, x, t)$ with arguments based on entropy admissibility criteria to select the physical solution. These arguments are well-known in the field of nonlinear conservation laws. By this strategy, we keep track of all the properties inherited from the physical velocity, in particular those regarding the boundary conditions. Finally, one emphasizes that, in the practical SPH formalism, the regularization by convolution of the physical velocity is of current use for Euler equations. The mathematical analysis of the resulting scheme is however a very open question.

In what follows, we start with a brief review of the SPH formalism.

2.1. Review of SPH. Historically, SPH was introduced at the end of the seventies by Lucy in [15] and Gingold and Monaghan in [20], as an alternative to classical methods (based on grid technique) to solve compressible Euler equations. The method still uses computational nodes called particles destined to be sprinkled through the 
domain, but does not require any pre-specified connectivity of these particles, or locally regular topological structure as it is needed for traditional meshing. Basically, the approximate solutions of equation (1) are computed for any time $t$ with respect to a set denoted by $K\left(K \subset \mathbb{Z}^{d}\right)$ of moving particles provided by a suitable quadrature formula $\left(x_{k}(t), w_{k}(t)\right)_{k \in K^{1}}$. In this formula, $x_{k}(t)$, which is the position of the particle $k \in K$ and $w_{k}(t)$ which is its effective weight, are solutions to the following systems of differential equations

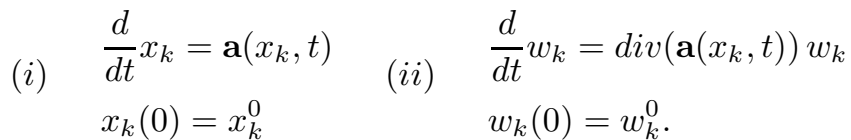

The quadrature formula $\left(x_{k}^{0}, w_{k}^{0}\right)_{k \in K}$ defines the initial particle distribution through the domain $\Omega$. The solutions of the system $(i)$ are the classical characteristic curves of the field $\mathbf{a}$, whereas those of equation (ii) reflect the evolution of the weights (i.e. the deformation of the particle distribution) in the change of coordinates. Thereby, the accuracy of the SPH approximation is first connected to the quadrature rule over $\Omega$

$$
\int_{\Omega} g(x) d x \approx \sum_{k \in K} w_{k} g\left(x_{k}\right)
$$

The available regularity of the field a makes it that this approximation is accurate for any $t>0$, as soon as it is accurate initially and the particles and their weights move according to (4). In most of practical computations, the particles are initially distributed on a regular grid (for instance cubic grid) and it is quite easy to find suitable weights such that the error in (5) is of "infinite order" (see Raviart [39]) when applied to $\mathcal{C}^{\infty}$ functions $g$ that vanish sufficiently rapidly at the infinity. In our setting, the set $K$ of the initial distribution of particles could be performed by using finite elements triangulation denoted by $P_{\Omega}$ and by considering that the particles are initially distributed on the center of the elements $B_{k}^{0} \in P_{\Omega}$ of this triangulation with their initial weights $w_{k}^{0}=$ meas $\left(B_{k}^{0}\right)$. If we denote by $B_{k}(t)$ (or simply $B_{k}$ by omiting the time dependance) the image at time $t$ of the cell $B_{k}^{0}$ by the vector field a such that meas $\left(B_{k}\right)=w_{k}$, then the $P^{1}$ finite element approximation gives

$$
\left|\mathcal{E}^{h}(g)\right| \leq C(\Omega) h \quad \text { with } \quad \mathcal{E}^{h}(g)=\sum_{k \in K} \mathcal{E}_{k}(g):=\sum_{k \in K}\left(\int_{B_{k}} g(x) d x-w_{k} g\left(x_{k}\right)\right) .
$$

The SPH method takes advantage of the particle distribution to provide by convolution, a discrete derivative operator denoted by $D_{\varepsilon}$ approximating first derivatives as follows

$$
\nabla g \approx D_{\varepsilon} g(x)=\sum_{k \in K} w_{k} g\left(x_{k}\right) \nabla \zeta^{\varepsilon}\left(x-x_{k}\right), \quad \zeta^{\varepsilon}(x)=1 / \varepsilon^{d} \zeta(x / \varepsilon),
$$

where the smooth function $\zeta$ is nonnegative with compact support and verifies

$$
\text { (i) } \int_{\mathbb{R}^{d}} \zeta(x) d x=1 \quad \text { (ii) } \int_{\mathbb{R}^{d}} x^{i} \zeta(x) d x=0 \quad(i \in 1, \ldots, d) .
$$

\footnotetext{
${ }^{1}$ in the sequel of this paper, we omit the time dependence when there is no ambiguity
} 
For the conservativity of the SPH scheme, it is convenient to work with a symmetrized version of $D_{\varepsilon}$ denoted by $D_{\varepsilon, s}$ (s: refers to this symmetry)

$$
D_{\varepsilon, s} g(x):=D_{\varepsilon} g(x)-g(x) . D_{\varepsilon} 1(x)
$$

and obtained by substituting the following vanishing error term

$$
g(x) . D_{\varepsilon} 1(x) \approx g(x) \nabla 1=0 .
$$

Accuracy results due to Raviart and Mas-Gallic are available in case of $\Omega=\mathbb{R}^{d}$ in ([39]) and ([32]). To make use of these results, one needs to restrict the validity of the approximations of (7) and (10) to the subset of $x \in \Omega$ such that $\operatorname{supp}\left(\zeta^{\epsilon}\left(x-x_{k}\right) \cap \partial \Omega=\right.$ $\emptyset$ for all $k \in K$. Thus, if one considers the subset $\Omega^{\kappa^{\prime}}=\{y \in \Omega$ : distance $(y, \partial(\Omega)) \geq$ $\left.\kappa^{\prime}>2 \varepsilon\right\}$, then, for all $\varphi \in W^{2, \infty}(\Omega)$ and for all $T>0$

$$
\left\|D_{\varepsilon} \varphi(x)-D \varphi(x)\right\|_{\infty} \leq C\left(T, \Omega^{\kappa^{\prime}}\right)\left(\varepsilon|\varphi|_{1, \infty}+\frac{h}{\varepsilon^{2}}\|\varphi\|_{2, \infty}\right), \quad \forall x \in \Omega^{\kappa^{\prime}} .
$$

In particular, this last estimate implies

$$
\left\|D_{\varepsilon} 1(x)\right\|_{\infty} \leq C\left(T, \Omega^{\kappa^{\prime}}\right) \frac{h}{\varepsilon^{2}}, \quad \forall x \in \Omega^{\kappa^{\prime}} .
$$

Therefore, the combination of (11) and (12) yields

$$
\forall x \in \Omega^{\kappa^{\prime}} \quad\left\|D_{\varepsilon, s} \varphi(x)-D \varphi(x)\right\|_{\infty} \leq C\left(T, \Omega^{\kappa^{\prime}}\right)\left(\varepsilon|\varphi|_{1, \infty}+\frac{h}{\varepsilon^{2}}\|\varphi\|_{2, \infty}\right) .
$$

Notice that, on account of the compact support of the cut-off $\zeta$ and the regularity of the field $\mathbf{a}$, a straightforward calculation gives the following estimates

$$
\begin{gathered}
\operatorname{card}\left\{k \in K ;\left\|\nabla \zeta^{\varepsilon}\left(x-x_{k}\right)\right\| \neq 0\right\} \leq C\left(\frac{\varepsilon}{h}\right)^{d} \quad(i i) \quad C_{1} h^{d} \leq w_{k} \leq C_{2} h^{d} \\
\left\|\nabla \zeta^{\varepsilon}\left(x-x_{k}\right)\right\| \leq \frac{C}{\varepsilon^{d+1}} \quad \text { (iv) } \quad \sum_{k \in K} w_{k}\left\|\nabla \zeta^{\varepsilon}\left(x-x_{k}\right)\right\| \leq \frac{C}{\varepsilon} .
\end{gathered}
$$

These estimates will be on a systematic use when evaluating errors terms following on our SPH scheme of equation (1).

Comments. It is apparent from the estimates (11-13) that in the SPH setting, the convergence of the approximations (7) and (10) is obtained by letting simultaneously $h \rightarrow 0, \varepsilon \rightarrow 0$ and $h / \varepsilon^{2} \rightarrow 0$ (which in the sequel will be denoted by $\Delta(\varepsilon, h) \rightarrow 0$ ). This clearly shows that the parameter discretization $h$ has to be taken much smaller than the smoothing lenght $\varepsilon$, precisely $h=o\left(\varepsilon^{2}\right)$. As a result, the ratio $v=\varepsilon / h$ becomes much bigger and tends to infinity. To show the central role of this resulting scaling $v$ in the SPH formalism, note that by (14) $(i)$, it provides the appropriate relatively constant number $N_{s p h}$ of the neighboring particles inside the smoothing lenght $\varepsilon$ of each particle so that the local approximation (7) makes sense. Moreover, the fact that $v$ tends to infinity can be seen as a necessary condition to make possible the passage limit from the discrete SPH model to the continuous fluid flow model. We refer to [26] for some convergence tests in the simulation of fracture analysis. We should point out that by (14) $(i), N_{s p h}$ also depends on the space dimension $d$. So, in practice, the parameters $h$ and $\varepsilon$ have to be chosen such that $N_{s p h}$ is around 25 for $d=2$ computations and 50 in $d=3$. 
2.2. Particle formulation of boundary conditions. The question of deriving an appropriate SPH scheme of equation (1) in unbounded domains is treated in [4]. Since the solution develops singularities in finite time, even with smooth initial data, the weak formulation of (1) is doubtless the right way to derive well-posed SPH schemes. Herein for "nice" test functions, this formulation reads

$$
\begin{aligned}
\int_{\Omega \times \mathbb{R}_{+}}\left(u \mathcal{L}_{\mathbf{a}}^{*} \varphi+F(u, x, t) \cdot \nabla \varphi+S(u, x, t) \varphi\right) d x d t & \\
& -\int_{\partial \Omega \times \mathbb{R}_{+}} F(u, x, t) \cdot n \varphi d \sigma(x) d t=0 .
\end{aligned}
$$

This clearly shows the remaining difficulty we have to deal with, which concerns the derivation of a suitable particle formulation of the boundary integral term by taking into account nonlinear effects at the boundary. To motivate the investigation of this question, recall that in the SPH formalism, the fluid flow is represented by fluid pseudo-particles. These individual particles interact with one another, moving with the flow and carrying with them all of the computational information about the fluid. Fluid properties are then interpolated between the particles. In other words, in the model equation (1) (see the scheme (26)), the term $\operatorname{div} F$ is interpreted as an internal volume force while the right hand side $S$ acts as an external volume force. Following these features, one needs to find a way to associate to the boundary contribution an appropriate volume approximation making relevant the interaction with the fluid particles and such that the necessary boundary conditions are satisfied. To this end, two approaches will be analysed. The first one is based on the so-called boundary particles and boundary forces. It was first used by Monaghan [33] in the simulation of gravity currents. Here, we deal with the derivation of a mathematical framework to reinforce the existing approach by extending it use to the numerical modeling of other types of physical phenomena such as solid friction and multiphase flows. Moreover, nonlinear effects at the boundary of equation (1) will be taken into account by solving appropriate Riemann problems. The stability of the resulting numerical model will be performed by using the equilibrium condition for a uniform field. The second approach is the well-known approach of ghost particles used to model specular reflection boundary conditions in case of plane boundaries. Herein, as for boundary forces approach, we deal with the treatment of general curved boundaries and provide under the same machinery an efficient numerical model to handle different types of boundary conditions including free boundaries (see [8]).

In what follows, we first focus on describing these two approaches for a given boundary data, while the question concerning nonlinear effects at the boundary will be treated in section 3 .

2.2.1. Boundary forces. Let $\beta$ a regular function of the real variable $y \in[0,1]$, such that

$$
(i), 0 \leq \beta(y), \quad(i i), \beta(y)=0, \quad \text { for } y \geq 1, \quad(i i i), \int_{0}^{1} \beta(y) d y=1 .
$$

Let also introduce in a neighborhood of $\partial \Omega$, the change of coordinates

$$
\begin{gathered}
\Omega \ni x \rightarrow(\bar{x}, y) \in \partial \Omega \times\left(-\kappa^{\prime}, 0\right) \\
\bar{x}=x-y n(\bar{x}), \quad \text { for some } \quad \kappa^{\prime}>0 .
\end{gathered}
$$


To perform the numerical model of boundary forces, one first considers a finite element type triangulation $E_{\partial \Omega}$ of the boundary $\partial \Omega$. To this triangulation, we associate a finite element interpolation $R_{E}(g)$ of any boundary function $g(\bar{x}, t)$

$$
R_{E}(g)(\bar{x}, t)=\sum_{i \in N_{E}} g\left(\bar{x}_{i}, t\right) \Psi_{i}(\bar{x})
$$

where the summation is taken over the degrees of freedom $N_{E}$ (respectively located at $\bar{x}_{i} \in \partial \Omega, i \in N_{E}$ ) of the finite element, associated with the basic polynomial functions $\Psi_{i}(\bar{x})$. These degrees of freedom, located on the boundary, can be considered either as boundary particles (moving or fixed particles) or as points of a fixed grid.

On the other hand, take $\varphi \in \mathcal{C}^{1}\left(\bar{\Omega} \times \mathbb{R}_{+}\right)$, then the change of variables gives

$$
\begin{aligned}
\int_{\partial \Omega} g(x, t) \varphi d \sigma(x) & =\lim _{\kappa^{\prime} \rightarrow 0} \int_{\partial \Omega} \int_{0}^{1} g(x(\bar{x}, 0), t) \beta(y) \varphi\left(x\left(\bar{x}, \kappa^{\prime} y\right), t\right) J_{\partial \Omega}(\bar{x}) d \bar{x} d y \\
& \left.=\lim _{\kappa^{\prime} \rightarrow 0} \int_{\Omega} g(x(\bar{x}, 0), 0), t\right) \beta^{\kappa^{\prime}}(y) \varphi(x, t) J_{\partial \Omega}(\bar{x}) J(x) d x,
\end{aligned}
$$

where the kernel $\beta^{\kappa^{\prime}}(y)=\frac{1}{\kappa^{\prime}} \beta\left(y / \kappa^{\prime}\right)$ and $J$ and $J_{\partial \Omega}$ are the Jacobian associated with the changes of coordinates. Consequently, for $\kappa^{\prime}$ sufficiently small,

$$
\int_{\partial \Omega} g(\bar{x}, t) \varphi d \sigma(x) \approx \int_{\Omega} g(\bar{x}, t) \beta^{\kappa^{\prime}}(y) \varphi(x, t) J_{\partial \Omega}(\bar{x}) J(x) d x .
$$

Therefore, an extension of $g(\bar{x}, t)$ to the whole domain $\Omega$ is given by

$$
g_{\kappa^{\prime}}(x(\bar{x}, y), t)=g(x(\bar{x}, 0), t) J_{\partial \Omega}(\bar{x}) J(x(\bar{x}, y)) \beta^{\kappa^{\prime}}(y) .
$$

Moreover, if one replaces $g(x(\bar{x}, 0), t)$ by its finite element interpolation $(17)$, one may write

$$
\begin{aligned}
& \int_{\Omega} g_{\kappa^{\prime}}(x, t) \varphi d x \underbrace{\approx}_{\text {by }(17)} \sum_{j \in N_{E}} g\left(\bar{x}_{j}, t\right) \int_{\Omega} J_{\partial \Omega}(\bar{x}) \Psi_{j}(\bar{x}) \beta^{\kappa^{\prime}}(y) J(x) \varphi d x \\
& \underbrace{\approx}_{\text {by }(5)} \sum_{k \in K, j \in N_{E}} w_{k} \varphi_{k} J\left(x_{k}\right) J_{\partial \Omega}\left(\bar{x}_{k}\right) g\left(\bar{x}_{j}, t\right) \Psi_{j}\left(\bar{x}_{k}\right) \beta^{\kappa^{\prime}}\left(y_{k}\right) .
\end{aligned}
$$

In term of external forces acting on the particles $k \in K$, this last formula can be interpreted by associating to each boundary particle (or grid point) $j$, a volume boundary force field $f_{j}(x)$ at the point $x$ defined by

$$
f_{j}(x(\bar{x}, y))=J(x) J_{\partial \Omega}(\bar{x}) g\left(\bar{x}_{j}\right) \Psi_{j}(\bar{x}) \beta^{\kappa^{\prime}}(y) .
$$

The resulting boundary force acting on each fluid particle $k \in K$ moving on the boundary layer $\left(-\kappa^{\prime}, 0\right) \times \partial \Omega$ is then given by

$$
f(x)=\sum_{j \in N_{T}} f_{j}(x)
$$

REMARK 2.1. The finite element approximation (17) is well-fitted when modeling physical problems as solid friction or when coupling SPH method with Eulerian methods in such a way that the best aspects of both approaches can be incorporated into a single model. We refer to [8] for more details. 
2.2.2. Ghost particles. This technique is formulated so that the local conservativity and consistency of the SPH scheme near the boundary are satisfied. These numerical requirements are achieved by generating outside the domain $\Omega$ a set of particles distribution called ghost particles. The physical properties of these particles are similar to the ones of fluid particles inside $\Omega$. To formulate this approach,one needs to construct an appropriate extension $\tilde{\Omega} \subset \mathbb{R}^{d}$ of the set $\Omega$. In this view, one considers the local system of coordinates $(\bar{x}, y)$ over $\partial \Omega \times\left(-\kappa^{\prime}, 0\right)$. Since the boundary is assumed to be smooth enough, then there exists a diffeomorphism $M$ which maps to any point $x=\bar{x}-y n(\bar{x}) \in \partial \Omega \times\left(-\kappa^{\prime}, 0\right)$ the point

$$
M(x)=\bar{x}+y n(\bar{x}) \in \Omega_{M}:=\partial \Omega \times\left(0, \kappa^{\prime}\right) .
$$

Thus, for each particle $k \in K$ of position $x_{k} \in K$, sufficiently close to the boundary $\partial \Omega$, one may associate a new particle called ghost particle located at the position $M\left(x_{k}\right)$. To get an appropriate quadrature formula over $\Omega_{M}$, one can take as a weight of the ghost particle the weight of the particle that it is ghost multiplied by $J_{M}\left(x_{k}\right)$ the Jacobian determinant $\left|\operatorname{det}\left(D M\left(x_{k}\right)\right)\right|$ at point $x_{k}$. Thereby, denoting by $G$ the set of these ghost particles that is

$$
G=\operatorname{card}\left\{k \in \mathbb{Z}^{d}, x_{k}(t) \in \Omega_{M}\right\},
$$

one provides a quadrature formula over $\tilde{\Omega}=\Omega \cup \Omega_{M}$ with its associate quadrature rule as follows

$$
\int_{\tilde{\Omega}} g(x) d x \approx \sum_{k \in K \cup G} \tilde{\omega}_{k}(t) g\left(x_{k}(t)\right)
$$

with

$$
\tilde{\omega}_{k}(t)=\left\{\begin{array}{l}
\omega_{k}(t) \text { if } x_{k} \in \Omega \\
\omega_{M^{-1}(k)}(t) J \circ M^{-1}\left(x_{k}(t)\right) \text { if } x_{k} \in \Omega_{M} .
\end{array}\right.
$$

The corresponding discrete derivative operator $\tilde{D}_{\varepsilon}$ is defined for any $g \in \mathcal{C}^{1}(\tilde{\Omega})$ by

$$
\forall x \in \Omega, \quad \tilde{D}_{\varepsilon} g(x)=\sum_{k \in K \cup G} \tilde{\omega}_{k}(t) g\left(x_{l}\right) \nabla \zeta^{\varepsilon}\left(x-x_{k}\right) .
$$

We also define

$$
\tilde{D}_{\varepsilon, s} g(x)=\tilde{D}_{\varepsilon} g(x)-g(x) \tilde{D}_{\varepsilon} 1(x) .
$$

Accuracy results (11-13) can therefore be extended to the whole domain $\Omega$ instead of $\Omega^{\kappa^{\prime}}$.

REMARK 2.2. There exists another technique to provide the local conservativity of SPH method called the semi-analytic approach (see [10]). It is based on the use of the exact values of the integrals of the shape function and its derivatives outside the domain $\Omega$ instead of generating a new distribution of ghost particles, by computing the integrals

$$
\int_{\mathbb{R}^{d} / \Omega} \zeta^{\varepsilon}(x-y) d y, \int_{\mathbb{R}^{d} / \Omega} \nabla \zeta^{\varepsilon}(x-y) d y .
$$


For the half-plane or polyhedral boundaries, one may compute these integrals in case of polynomial shape functions $\zeta$ by using formal calculus computer codes such as the results given by Herand in [18].

REMARK 2.3. Note that, in practice, the technique of ghost particles needs the creation of a ghost particle's distribution at any time $t$, so that the number of particles inside the radius $\varepsilon$ is equal to the constant value $N_{\text {sph }}$ for all the particles near the boundary $\partial \Omega$. However, for the boundary forces' technique, the boundary particles are set initially.

3. Weak form of the SPH scheme. In this section, we make use of the results of the previous section to perform the SPH scheme of equation (1).

3.1. Case of boundary forces. We proceed into three steps. First, we derive the particle scheme in the interior domain. Secondly, we make use of the upwind particle scheme developed in ([4]), to fix the problem of the stability of the scheme. The third step will be devoted to provide the boundary contribution.

Step 1 (The interior domain). Define the adjoint operator $D_{\varepsilon, s}^{*}$ of $D_{\varepsilon, s}$ according to the $L^{2}$ discrete scalar product as

$$
\left(D_{\varepsilon, s} \varphi, \Psi\right)_{h}=-\left(\varphi, D_{\varepsilon, s}^{*} \Psi\right)_{h} \quad(\varphi, \Psi)_{h}:=\sum_{i \in K} w_{i} \varphi_{i} . \Psi_{i}
$$

Consider also the hybrid particle approximation $\bar{u}_{h}(x, t)$ introduced in ([4]) in order to extend finite volume techniques to the particle scheme. It is defined by

$$
\bar{u}_{h}(x, t)=\sum_{k \in K} u_{k} \chi_{B_{k}}(x),
$$

where $u_{k}$ stands for an approximation of the exact solution of problem (1) and $\chi_{B_{k}}$ is the characteristic function of the set $B_{k}$. To get the particle scheme of equation (1) inside $\Omega$, let us take the following weak model $\left(\kappa=O\left(\kappa^{\prime}\right)\right)$

$$
\int_{[0, T]}\left[\left(\bar{u}_{h}, \mathcal{L}_{a}^{*}(\varphi)\right)_{h}+\left(F\left(\bar{u}_{h}, x, t\right), \chi^{\kappa} D_{\varepsilon, s} \varphi\right)_{h}+\left(S\left(\bar{u}_{h}, x, t\right), \varphi\right)_{h}\right] d t=0,
$$

where $\varphi$ is a test function and $\chi^{\kappa}$ is a suitable regularization of the characteristic function $\chi$ of the domain $\Omega$, for instance

$$
\chi^{\kappa}(x(\bar{x}, y))= \begin{cases}0 & 0 \leq y<\kappa \\ 1 / 2+3(y-2 \kappa) /(4 \kappa)-(1 / 4)((y-2 \kappa) / \kappa)^{3} & \kappa \leq y<3 \kappa \\ 1 & y \geq 3 \kappa\end{cases}
$$

In view of (22) together with an integration by parts with respect to $t$, one easily proves that (24) is equivalent to the system of differential equations

$$
\forall k \in K, \quad \frac{d}{d t}\left(w_{k} u_{k}\right)+w_{k}\left(D_{\varepsilon, s}^{*}\right)\left(\chi^{\kappa} F\right)_{x=x_{k}}=w_{k} S\left(u_{k}, x_{k}, t\right),
$$

where

$$
D_{\varepsilon, s}^{*}\left(\chi^{\kappa} F\right)_{x=x_{k}}=\sum_{l \in K} w_{l}^{n}\left(\chi_{k}^{\kappa} F\left(u_{k}, x_{k}, t\right) \mathcal{A}_{k l}-\chi_{l}^{\kappa} F\left(u_{l}, x_{l}, t\right) \mathcal{A}_{l k}\right)
$$


and the abbreviation $\mathcal{A}_{i j}=\left(\nabla \zeta^{\varepsilon}\right)_{x=x_{i}}\left(x_{i}-x_{j}\right)$ has been used.

Note that the weak consistency of the resulting numerical scheme (26) is obtained from (24) by a direct application of the quadrature error (6) and the accuracy result (13). The global conservativity is however acquired by the use of the derivative operator $D_{\varepsilon, s}$ defined in (9), instead of $D_{\varepsilon}$. Indeed, without the boundary contribution if we take in the scheme (26), the sum over $k \in K$, then we get

$$
\frac{d}{d t}\left(\sum_{k \in K} w_{k} u_{k}\right)+\sum_{k \in K} w_{k} D_{\varepsilon, s}^{*}\left(\chi^{\kappa} F\right)_{x=x_{k}}=\sum_{k \in K} w_{k} S\left(u_{k}, x_{k}, t\right) .
$$

This is nothing but the discrete equivalent inside $\Omega$ of

$$
\frac{d}{d t} \int_{\Omega} u(x, t) d x=\int_{\Omega} S(u(x, t), x, t) d x
$$

due to the fact that $\sum_{k \in K} w_{k} D_{\varepsilon, s}^{*}\left(\chi^{\kappa} F\right)_{x=x_{k}}=0$. By symmetry, this last point can be seen by switching the indices $k$ and $l$ in one of the two terms composing it.

Note in passing that the general form of scheme (26) is well-defined for all known formulations of SPH developed for Euler equations to remedy the problem of particles distortion. In these formulations, the smoothing length $\varepsilon$ is considered as an adaptive function $\varepsilon(x)$ in order to keep the number of particles inside the smoothing radius $\varepsilon\left(x_{k}\right)$ for all $k \in K$ is equal to a suitable constant value $N_{s p h}$. For instance, Gather and Scatter approximations, where respectively in the scheme $(26) \mathcal{A}_{i j}=\left(\nabla \zeta^{\varepsilon\left(x_{i}\right)}\right)\left(x_{i}-x_{j}\right)$ and $\mathcal{A}_{i j}=\left(\nabla \zeta^{\varepsilon\left(x_{j}\right)}\right)\left(x_{i}-x_{j}\right)$ or the symmetric version in which $\varepsilon=\left(\varepsilon\left(x_{i}\right)+\varepsilon\left(x_{j}\right)\right) / 2$. This last formulation is the most popular of them (see [33], [19], [20], [34]).

Step 2 (The upwind particle scheme). As it has already been underlined in ([4], [6]), the scheme (26) is somehow a generalized finite difference centered scheme, well known to be unconditionally unstable whenever a time-explicit discretization is used. To lift this difficulty, we have developed an original approach using nonlinear upwinding and Riemann approximate solvers well known in the field of finite difference schemes for nonlinear hyperbolic equations (see for instance [16], [17]). Indeed, the form of the scheme (26) computing the interaction between any pair of particles $(k, l)$ along the direction $n_{k l}$ connecting $x_{k}$ with $x_{l}$, suggests the introduction at the point $x_{k l}=\left(x_{k}+x_{l}\right) / 2$ of the Riemann problem

$$
\left\{\begin{array}{l}
\frac{\partial}{\partial t}(u)+\frac{\partial}{\partial x}\left(F\left(u, x_{k l}, t\right) . n_{k l}\right)=0, \quad \text { with } n_{k l}=\mathcal{A}_{k l} /\left\|\mathcal{A}_{k l}\right\| . \\
u(x, 0)=\left\{\begin{array}{l}
u_{k} \text { if } x<0 \\
u_{l} \text { if } x>0 .
\end{array}\right.
\end{array}\right.
$$

Therefore, a suitable approximation of such a problem can be performed by introducing a 1-dimensional finite difference scheme $g$ in a conservation form associated to (29). This numerical scheme $g$ is consistent with the nonlinearity $F . n(x)$ and conservative, i.e $g(n(x), u, u)=F(u, x, t) \cdot n(x)$ and $g(n, u, v)+g(-n, v, u)=0$ respectively. The corresponding numerical viscosity $Q(n(x), u, v)$ and the incremental coefficient $C(n(x), u, v)$ are then classically defined by

$$
\begin{aligned}
& Q(n(x), u, v)=\frac{F(x, t, u) \cdot n(x)-2 g(n(x), u, v)+F(x, t, v) \cdot n(x)}{v-u} \\
& C(n(x), u, v)=\frac{F(u, x, t) \cdot n(x)-g(n(x), u, v)}{v-u} .
\end{aligned}
$$


Thus, the upwind numerical scheme which consists in finding functions $t \in \mathbb{R}^{+} \longrightarrow$ $u_{k}(t) \in \mathbb{R}, k \in K$, reads

$$
\frac{d}{d t}\left(w_{k} u_{k}\right)+w_{k} \sum_{l \in K} w_{l}\left(\chi_{k}^{\kappa} g\left(n_{k l}, u_{k}, u_{l}\right)\left\|\mathcal{A}_{k l}\right\|-\chi_{l}^{\kappa} g\left(n_{l k}, u_{l}, u_{k}\right)\left\|\mathcal{A}_{l k}\right\|\right)=w_{k} S_{k} .
$$

As numerical fluxes $g$ for such an upwinding, Lax Friedrich and Godunov schemes are well-fitted. These schemes are in fact monotone finite difference schemes (see Crandall and Majda [12] and Kuznetsov and Volosin [25]) which belong to the widest class of E-schemes (Osher [36]). In the following, we suppose that $g$ is an E-scheme.

Step 3 (The boundary contribution). Let $b_{\kappa^{\prime}}(x, t)$ be the extension to $\Omega$ of the boundary data $b(\bar{x}, t)$ according to (18). In our model of boundary forces the boundary contribution will be computed in the boundary layer $\left[-\kappa^{\prime}, 0\right] \times \partial \Omega$. Thus, for each particle $k \in K$ of position $x_{k}$ moving in $\left[-\kappa^{\prime}, 0\right] \times \partial \Omega$, one considers additionally to the Riemann problem (29) the following one at $x_{k}$ along the direction $\tilde{n}_{k}$ (to be determined a posteriori)

$$
\left\{\begin{array}{l}
\frac{\partial}{\partial t}(v)+\frac{\partial}{\partial x}\left(F\left(v, x_{k}, t\right) \cdot \tilde{n}_{k}\right)=0 \\
v(x, 0)=\left\{\begin{array}{l}
u_{k} \text { if } x<0 \\
b_{k}=b_{\kappa^{\prime}}\left(x_{k}\right) \text { if } x>0 .
\end{array}\right.
\end{array}\right.
$$

Accordingly, one may use the numerical scheme $g$ to approximate this Riemann problem. Moreover, for the stability of the scheme, one needs to introduce a function $\theta(x)$ (also to be computed) with $\operatorname{supp}(\theta) \subset\left(-\kappa^{\prime}, 0\right) \times \partial \Omega$ so that the global upwind scheme reads

$$
\begin{gathered}
\frac{d}{d t}\left(w_{k} u_{k}\right)+w_{k} \sum_{l \in K} w_{l}\left(\chi_{k}^{\kappa} g\left(n_{k l}, u_{k}, u_{l}\right)\left\|\mathcal{A}_{k l}\right\|-\chi_{l}^{\kappa} g\left(n_{l k}, u_{l}, u_{k}\right)\left\|\mathcal{A}_{l k}\right\|\right) \\
+w_{k} \theta\left(x_{k}\right) g\left(\tilde{n}_{k}, u_{k}, b_{k}\right)=w_{k} S_{k} .
\end{gathered}
$$

Thus, the scheme (31) is well-posed if particularly the equilibrium condition for a uniform field is satisfied (i.e. with $F(u, x, t)=$ cte and $S(u, x, t)=0$ ). Thus, (31) reads

$$
\begin{aligned}
\frac{d}{d t}\left(w_{k} u_{k}\right)=0 \Longrightarrow \theta_{k} \tilde{n}_{k} & =-\sum_{l \in K} w_{l}\left(\chi_{k}^{\kappa} \mathcal{A}_{k l}-\chi_{l}^{\kappa} \mathcal{A}_{l k}\right) \\
& =-\left(D_{\varepsilon, s}^{*} \chi^{\kappa}\right)_{x=x_{k}} \equiv-D_{\varepsilon, s}^{*} \chi_{k}^{\kappa},
\end{aligned}
$$

which yields the following suitable choice

$$
\tilde{n}_{k}=-D_{\varepsilon, s}^{*} \chi_{k}^{\kappa} /\left\|D_{\varepsilon, s}^{*} \chi_{k}^{\kappa}\right\|, \quad \theta_{k}:=\theta\left(x_{k}\right)=\left\|D_{\varepsilon, s}^{*} \chi_{k}^{\kappa}\right\| .
$$

Therefore,

$$
\theta(x)=\left\{\begin{array}{lc}
\left\|D_{\varepsilon, s}^{*} \chi^{\kappa}(x)\right\| & \text { if } x \in\left[-\kappa^{\prime}, 0\right] \times \partial \Omega \\
0 & \text { elsewhere. }
\end{array}\right.
$$

To show the weak consistency with the boundary integral term in the model (31), one may take for simplicity the case of symmetry shaped function $\zeta(x)\left(\right.$ i.e. $\left.\mathcal{A}_{k l}=-\mathcal{A}_{l k}\right)$. Thus, on the one hand, the accuracy result (11) gives

$$
-\left(D_{\varepsilon, s}^{*} \chi^{\kappa}\right)(x) \underset{\Delta(h, \varepsilon) \rightarrow 0}{\longrightarrow} \nabla\left(1-\chi^{\kappa}\right)(x), \quad \text { in } \quad L_{l o c}^{\infty}(\Omega) .
$$


On the other hand, one replaces the numerical flux $g\left(\tilde{n}_{k}, u_{k}, b_{k}\right)$ by its expression from (30) and take the contribution coming from the boundary forces, then a straightforward calculation using (33) and the quadrature rule (6) gives (with the choice $\kappa^{\prime}=3 \kappa$ )

$$
\begin{aligned}
& \lim _{\kappa^{\prime} \rightarrow 0 \Delta(h, \varepsilon) \rightarrow 0} \lim _{k \in K}-\left(\sum_{k} w_{k} \varphi_{k} F\left(b_{\kappa^{\prime}}\left(x_{k}, t\right), x_{k}, t\right) D_{\varepsilon, s}^{*} \chi_{k}^{\kappa}\right) \\
& =\lim _{\kappa^{\prime} \rightarrow 0} \int_{\Omega} F\left(b_{\kappa^{\prime}}(x, t), x, t\right) \nabla\left(1-\chi^{\kappa}\right)(x) \varphi(x) d x=\int_{\partial \Omega} F(b, x, t) . n \varphi d \sigma(x) .
\end{aligned}
$$

Note however that, due to the non smoothness of the hybrid particle approximation $\bar{u}_{h}$, the evaluation of the other terms requires more sophisticated arguments. These arguments use the concept of measure-valued solutions and the convergence for measures in the weak topology $\sigma\left(M_{b}, \mathcal{C}_{c}\right)\left(M_{b}\right.$ denotes the set of bounded Radon measures).

In the sequel, to make the reading easier, we consider that the cut off function $\zeta(x)$ is symmetric, in which case the scheme (31) reads

$$
\frac{d}{d t}\left(w_{k} u_{k}\right)+w_{k} \sum_{l \in K} w_{l}\left(\chi_{k}^{\kappa}+\chi_{l}^{\kappa}\right) g\left(n_{k l}, u_{k}, u_{l}\right)\left\|\mathcal{A}_{k l}\right\|+w_{k} \theta\left(x_{k}\right) g\left(\tilde{n}_{k}, u_{k}, b_{k}\right)=w_{k} S_{k} .
$$

Moreover, for the convenience, one may replace the average $\left(\chi_{k}^{\kappa}+\chi_{l}^{\kappa}\right) / 2$ by the value $\chi^{\kappa}\left(x_{k l}\right)$ at the mean point $x_{k l}$ denoted by $\chi_{k l}^{\kappa}$ to get

$$
\frac{d}{d t}\left(w_{k} u_{k}\right)+w_{k} \sum_{l \in K} w_{l} \chi_{k l}^{\kappa} 2 g\left(n_{k l}, u_{k}, u_{l}\right)\left\|\mathcal{A}_{k l}\right\|+w_{k} \theta\left(x_{k}\right) g\left(\tilde{n}_{k}, u_{k}, b_{k}\right)=w_{k} S_{k} .
$$

Finally, using the forward Euler scheme in time, we get the following algorithm

(i) $\quad u_{k}^{0}=\frac{1}{\operatorname{meas}\left(B_{k}\right)} \int_{B_{k}} u(x, 0) d x$

$$
\begin{aligned}
& \tilde{u}_{k}^{n+1}=u_{k}^{n}-\tau^{n} 2 \sum_{l \in K} w_{l}^{n} \chi_{k l}^{\kappa} g\left(n_{k l}, u_{k}^{n}, u_{l}^{n}\right)\left\|\mathcal{A}_{k l}^{n}\right\| \\
& \quad-\tau^{n}\left(\theta_{k}^{n} g\left(\tilde{n}_{k}, u_{k}^{n}, b_{k}^{n}\right)-S_{k}^{n}\right)
\end{aligned}
$$

$$
\frac{w_{k}^{n+1}}{w_{k}^{n}} u_{k}^{n+1}=\tilde{u}_{k}^{n+1} .
$$

In the above algorithm, the position and the effective weight of any particle $k \in K$ are computed by integrating in (4) the system $(i)$ and the equation (ii) i.e.

$$
x_{k}^{n}=x_{k}\left(t^{n}\right) \quad w_{k}^{n}=w\left(x_{k}\left(t^{n}\right)\right) .
$$

In particular, we have the following relation connecting $w_{k}^{n+1}$ with $w_{k}^{n}$

$$
w_{k}^{n+1}=w_{k}^{n} \exp \left(\int_{t^{n}}^{t^{n+1}} \operatorname{div}\left(a\left(x_{k}(t), t\right) d t\right):=w_{k}^{n} D a_{k}^{n} .\right.
$$

REMARK 3.1. One emphasizes that our analysis can be done for the general case of an adaptive smoothing length $\varepsilon(x)$ subject to a slight adaptation of Raviart's approximation results (11) (see [39], [32]), together with the additional bound $C_{1} \leq$ $\varepsilon(x) / \varepsilon_{0} \leq C_{2}$. For more details on the derivation of these approximation results, we refer to [26] or [7]. 
3.2. Case of ghost particles. Equipped with the quadrature formula over $\tilde{\Omega}$ (20) and the diffeomorphism $M$ (19) defined in section 2.2.2, one may extend the flux $F(u, x, t)$ and the boundary data $b(x, t)$ outside to $\Omega$ in $\Omega_{M}=\partial \Omega \times\left(0, \kappa^{\prime}\right)$ in the following appropriate way

$$
\Omega_{M} \ni x=\bar{x}+y n(\bar{x}) \longrightarrow\left\{\begin{array}{l}
F(u, x, t)=F\left(u, M^{-1}(x), t\right) \\
b(x, t)=b(\bar{x}, t),
\end{array}\right.
$$

with $M^{-1}(x)=\bar{x}-y n(\bar{x}) \in \partial \Omega \times\left(-\kappa^{\prime}, 0\right)$.

Let also denote by $\tilde{D}_{\varepsilon, s}^{*}$ the adjoint operator associated to $\tilde{D}_{\varepsilon, s}$ provided by $(21)$ in which the scalar product $(22)$ is taken over $\tilde{\Omega}$. For the wellposedness of the model below, we also take $\bar{u}_{h}(x, t)=b(x, t)$ for $x \in \Omega_{M}$. So, the particle model of (1) using the ghost particles' approach is given by

$$
\int_{[0, T]}\left[\left(\bar{u}_{h}, \mathcal{L}_{a}^{*} \varphi\right)_{h}-\left(\tilde{D}_{\varepsilon, s}^{*} F\left(\bar{u}_{h}, x, t\right)-S\left(\bar{u}_{h}, x, t\right), \varphi\right)_{h}\right] d t=0 .
$$

An integration by parts with respect to $t$ shows that this model consists in finding the sequence $t \longrightarrow u_{k}$ for all $k \in K$ solution of the system of differential equations

$$
\begin{aligned}
\frac{d}{d t}\left(w_{k} u_{k}\right) & +w_{k} \sum_{l \in K} w_{l}\left(F\left(u_{k}, x_{k}, t\right)+F\left(u_{l}, x_{l}, t\right)\right) \mathcal{A}_{k l} \\
& +w_{k} \sum_{l \in G} \tilde{w}_{l}\left(F\left(u_{k}, x_{k}, t\right)+F\left(b_{l}, x_{l}, t\right)\right) \mathcal{A}_{k l}=w_{k} S_{k} .
\end{aligned}
$$

In this scheme, the term in the right-hand side of (41) represents the SPH formulation of $\operatorname{div} F$ in the interior domain while the term in the left-hand side of (42) provides the suitable volume formulation associated with the boundary contribution $F . n$ in the weak sense (see Appendix A for the proof of this last fact in case of smooth solutions).

Using the numerical scheme $g$ introduced in the previous case, then the upwind particle scheme may be written

$$
\begin{aligned}
\frac{d}{d t}\left(w_{k} u_{k}\right) & +w_{k} \sum_{l \in K} w_{l} 2 g\left(n_{k l}, u_{k}, u_{l}\right)\left\|\mathcal{A}_{k l}\right\| \\
& +w_{k} \sum_{l \in G} \tilde{w}_{l} 2 g\left(n_{k l}, u_{k}, b_{l}\right)\left\|\mathcal{A}_{k l}\right\|=w_{k} S_{k} .
\end{aligned}
$$

Hence, using an explicit-time discretization, the numerical particle scheme is then given by

$$
\begin{aligned}
& u_{k}^{0}=\frac{1}{\operatorname{meas}\left(B_{k}\right)} \int_{B_{k}} u(x, 0) d x \\
& (i i)^{\prime} \quad \tilde{u}_{k}^{n+1}=u_{k}^{n}-\tau^{n} 2 \sum_{l \in K} w_{l}^{n} g\left(n_{k l}, u_{k}^{n}, u_{l}^{n}\right)\left\|\mathcal{A}_{k l}^{n}\right\| \\
& -\tau^{n} \sum_{l \in G} \tilde{w}_{l}^{n} 2 g\left(n_{k l}, u_{k}^{n}, b_{l}^{n}\right)\left\|\mathcal{A}_{k l}^{n}\right\|+\tau^{n} S_{k}^{n}
\end{aligned}
$$

(iii) $\quad \frac{w_{k}^{n+1}}{w_{k}^{n}} u_{k}^{n+1}=\tilde{u}_{k}^{n+1}$,

where the positions and the effective weights $\left(x_{k}^{n}, w_{k}^{n}\right)$ of the fluid particles $k \in K$ are computed by (37) and (38). 
4. Statement of the main results. We are now ready to state the convergence results of the two approximate solutions given by the schemes (36) and (45). To that purpose, let us denote by $\tau^{+}=\max _{t^{n} \leq T} \tau^{n}$ and $\tau^{-}=\min _{t^{n} \leq T} \tau^{n}$ and by (FS) and (DFS) the following assumptions

$$
\begin{aligned}
F & \in\left[\mathcal{C}\left(\mathbb{R} \times \Omega \times \mathbb{R}_{+}\right)\right]^{d}, \quad F(u, . . .) \in\left[\mathcal{C}^{1}\left(\Omega \times \mathbb{R}_{+}\right)\right]^{d} \\
S & \in \mathcal{C}\left(\mathbb{R} \times \Omega \times \mathbb{R}_{+}\right),
\end{aligned}
$$

$$
F^{i}(0, ., .), \partial_{x_{j}} F^{i}(0, . . .), S(0, . . .) \in L^{1}\left(\Omega \times \mathbb{R}^{+}\right) \text {. for } 1 \leq i, j \leq d
$$

THEOREM 4.1. Assume that the assumptions (FS) and (DFS) hold and that the flux $F$ and $S$ are Lipschitz with respect to $u$ uniformly on $(x, t)$. Suppose also that the initial and boundary data $\left(u_{0}, b\right)$ belong to $L^{\infty}(\Omega) \times L^{\infty}\left(\partial \Omega \times \mathbb{R}^{+}\right)$. Let $\bar{u}_{h}(x, t)$ be approximate solutions of (1), defined by (23) and computed either by the scheme (36) or by (45). Suppose in addition that $\kappa^{\prime}=O(\varepsilon)$ and that the following $C F L$ condition is satisfied for some constant $\beta, 0<\beta<1$

$$
\tau^{+}=(1-\beta) \underset{\substack{|u|,|v| \leq C_{0} \\ k, l \in K}}{\operatorname{Sup}} \frac{\varepsilon}{\left|C\left(n_{k l}, u, v\right)\right|},
$$

where the constant $C_{0}=C\left(T,\left\|u_{0}\right\|_{\infty},\|b\|_{\infty}\right)$. Then $\bar{u}_{h}(x, t)$ converges in $L_{l o c}^{p}\left(\Omega \times \mathbb{R}^{+}\right)$ (for $1 \leq p<\infty)$ towards $u$, the unique weak entropy solution of (1) in Otto's sense $[37]$, when $\Delta(\varepsilon, h) \rightarrow 0, \frac{\varepsilon}{\sqrt{\tau_{-}}} \rightarrow 0$ and $\kappa^{\prime} \rightarrow 0$.

In this convergence result, the additional assumption $\varepsilon=o\left(\sqrt{\tau_{-}}\right)$is used to get the suitable control of the global dissipation of the scheme (see [4] for more details in case $\Omega=\mathbb{R}^{d}$ ).

The proof of this theorem will be given in the case of boundary forces. The case of ghost particles can be done in a similar way (see [6] for the detailed proof). For both cases, the proof is based on the use of the concept of measure valued solutions (based on Young measures) and their uniqueness for equation (1). To define this concept of solutions for equation (1), let us consider, by the end of this section (for an easy presentation), that the transport field $\mathbf{a} \equiv 0$, since it does not play here any special role, except involving additional terms that can be included in the flux $F$.

Definition 4.2. A Young measure $\nu$, with its trace $\gamma \nu$ (see lemma 1.1 in [41]), is a measure solution to problem (1-3) if for any entropy-entropy flux pair $(\eta(u), \hbar(u, x, t))$ such that $\partial_{u} H^{i}(u, x, t)=\eta^{\prime}(u) \partial_{u} F^{i}(u, x, t)$ and for all nonnegative test function $\varphi \in \mathcal{C}_{c}^{1}\left(\bar{\Omega} \times \mathbb{R}_{+}\right)$,

$$
\begin{aligned}
& \int_{\Omega \times \mathbb{R}_{+}}\left\{\left\langle\nu_{x, t}(\lambda), \eta(\lambda)\right\rangle \partial_{t} \varphi+\left\langle\nu_{x, t}(\lambda), \hbar(\lambda, x, t)\right\rangle \nabla_{x} \varphi\right\} d x d t \\
& +\int_{\Omega \times \mathbb{R}_{+}}\left\langle\nu_{x, t}(\lambda), \sum_{i=1, d}\left(\partial_{x^{i}}\left(H^{i}(\lambda, x, t)\right)-\eta^{\prime}(\lambda) \partial_{x^{i}} F^{i}(\lambda, x, t)\right)\right\rangle \varphi d x d t \\
& +\int_{\Omega \times \mathbb{R}_{+}}\left\langle\nu_{x, t}(\lambda), \eta^{\prime}(\lambda) S(\lambda, x, t)\right\rangle \varphi d x d t \\
& \quad-\int_{\partial \Omega \times \mathbb{R}_{+}}\left\langle\gamma \nu_{s, t}(\lambda), B(\lambda, b, s, t)\right\rangle \cdot n(s) \varphi(s, t) d s d t \geq 0,
\end{aligned}
$$


and with

$$
\lim _{t \rightarrow 0+} \int_{\Omega}\left\langle\nu_{(x, t)}(\lambda),\left|\lambda-u_{0}(x)\right|\right\rangle d x=0
$$

The boundary entropy flux is defined by

$$
B(\lambda, b, s, t)=\hbar(b(s, t), s, t)-\eta^{\prime}(b(s, t))(F(b(s, t), s, t)-F(\lambda, s, t)) .
$$

REMARK 4.3. Note that the well-known boundary formulation introduced by Bardos, LeRoux and Nedelec in [1] using the Kruskov entropy |. - c| can be recovered from the above one (49) by taking $\eta^{\prime}()=.\operatorname{sgn}(.-c)$ to get

$$
B(\lambda, b, s, t)=\operatorname{sgn}(b-c)(F(\lambda, s, t)-F(c, s, t)) .
$$

However, the inequality (47) is not well-defined with the Kruskov entropy-entropy flux pair

$$
(|\lambda-c|, q(\lambda, c, x, t)=\operatorname{sgn}(\lambda-c)(F(\lambda, x, t)-F(c, x, t))) .
$$

This comes from the fact that for example the term

$$
\left\langle\nu_{x, t}(\lambda), \eta^{\prime}(\lambda) S(\lambda, x, t)\right\rangle=\left\langle\nu_{x, t}(\lambda), \operatorname{sgn}(\lambda-c) S(\lambda, x, t)\right\rangle
$$

is not well-defined for discontinuous functions. To remedy this problem, we consider a regularized entropy-entropy flux pair $\left(\eta^{\delta}(\lambda-c), H^{\delta}(\lambda, c, x, t)\right.$ of (50) provided by regularizing the sgn function

$$
\operatorname{sgn}_{\delta}(x)=\left\{\begin{array}{ll}
1 & \text { if } x \geq \delta \\
\frac{x}{\delta} & \text { if }-\delta<x<\delta \\
-1 & \text { if } x \leq-\delta
\end{array} \quad\left(\eta^{\delta}\right)^{\prime}(\lambda-c)=\operatorname{sgn}_{\delta}(\lambda-c),\right.
$$

then

$$
H^{\delta}(\lambda, c, x, t)=\int_{c}^{\lambda} \eta_{\delta}^{\prime}(v) \partial_{v} F(v, x, t) d v=q^{\delta}(\lambda, c, x, t)+q_{r}^{\delta}(\lambda, c, x, t)
$$

with

$$
\begin{aligned}
& q^{\delta}(\lambda, c, x, t)=\operatorname{sgn}_{\delta}(\lambda-c)(F(\lambda, x, t)-F(c, x, t)) \\
& q_{r}^{\delta}(\lambda, c, x, t)=\int_{c}^{\lambda}\left(\operatorname{sgn}_{\delta}(v-c)-\operatorname{sgn}_{\delta}(\lambda-c)\right) \partial_{v} F(v, x, t) d v .
\end{aligned}
$$

Moreover, a direct computation proves that

$$
\left|q_{r}^{\delta}(\lambda, c, x, t)\right| \leq C \delta
$$

Thus

$$
\left(\eta^{\delta}(\lambda-c), H^{\delta}(\lambda, c, x, t) \longrightarrow(|\lambda-c|, q(\lambda, c, x, t)) \quad \text { a.e } \quad \text { in } \quad \Omega \times \mathbb{R}^{+}\right. \text {. }
$$


TheOREM 4.4. Assume that $\left(u_{0}, b\right)$ belongs to $L^{\infty}(\Omega) \times L^{\infty}\left(\partial \Omega \times \mathbb{R}^{+}\right)$, that the assumption (FS) holds and that $\nu$ and $\sigma$ are Young measure solutions to (1-3), in the sense of definition (4.2), then, the inequality

$$
\begin{aligned}
\partial_{t} \int_{\Omega}\left\langle\nu_{x, t}(\lambda)\right. & \left.\otimes \sigma_{x, t}(\mu),|\lambda-\mu|\right\rangle d x \\
& \leq-\int_{\Omega}\left\langle\nu_{x, t}(\lambda) \otimes \sigma_{x, t}(\mu), \operatorname{sgn}(\lambda-\mu)(S(\lambda, x, t)-S(\mu, x, t))\right\rangle d x
\end{aligned}
$$

holds in the distribution sense on $\mathbb{R}_{+}$. If, in addition, $\nu$ and $\sigma$ satisfy the same initial condition (48) and $S$ is Lipschitz with respect to $u \in \mathbb{R}$ uniformly on $(x, t)$, then there exists a unique solution $u \in L^{\infty}\left(\Omega \times \mathbb{R}_{+}\right)$such that

$$
\nu_{y}=\sigma_{y}=\delta_{u(y)}, \quad \text { for a.e. } y \in \Omega \times \mathbb{R}_{+}
$$

and $u$ is the unique weak entropy solution to (1-3) in the sense of Otto [37].

Recall that the measure tensor product $\nu_{y} \otimes \sigma_{y}$ is defined for all $g \in \mathcal{C}\left(\mathbb{R}^{2}\right)$ by

$$
\left\langle\nu_{y} \otimes \sigma_{y}, g(\lambda, \mu)\right\rangle \equiv \int_{\mathbb{R}} \int_{\mathbb{R}} g(\lambda, \mu) d \nu_{y}(\lambda) d \sigma_{y}(\mu) .
$$

This uniqueness result is established in [3] in case where $F(u, x, t)=f(u)$ and $S(u, x, t)=0$. The proof of the present general result is a slight adaptation of the one of the previous case combined with Gronwall lemma. The main difficulty lies in the treatment of boundary conditions, which is completely fulfilled in [3].

We next give an equivalent definition to (4.2) which is well-fitted for the analysis of the scheme (36) since it requires less information than (4.2) for the weak formulation of boundary conditions. The proof of this equivalence is postponed to the end of the paper (Appendix B).

Definition 4.5. A Young measure $\nu$ is a measure solution to problem (1-3) if and only if

(In the interior domain) For any entropy-entropy flux pair $(\eta(u), \hbar(u, x, t))$ and $\forall \varphi \in \mathcal{C}_{c}^{1}\left(\Omega \times \mathbb{R}_{+}\right)$

$$
\mathcal{M}^{\eta}\left(\nu_{x, t}, \varphi\right) \geq 0
$$

with

$$
\begin{gathered}
\mathcal{M}^{\eta}\left(\nu_{x, t}, \varphi\right):=\int_{\Omega \times \mathbb{R}_{+}}\left\{\left\langle\nu_{x, t}(\lambda), \eta(\lambda)\right\rangle \partial_{t} \varphi+\left\langle\nu_{x, t}(\lambda), \hbar(\lambda, x, t)\right\rangle \nabla_{x} \varphi d x d t+\right. \\
\quad+\int_{\Omega \times \mathbb{R}_{+}}\left\langle\nu_{x, t}(\lambda), \sum_{i=1, d}\left(\partial_{x^{i}} H^{i}(\lambda, x, t)-\eta^{\prime}(\lambda) \partial_{x^{i}} F^{i}(\lambda, x, t)\right)\right\rangle \varphi d x d t \\
\quad+\int_{\Omega \times \mathbb{R}_{+}}\left\langle\nu_{x, t}(\lambda), S(\lambda, x, t)\right\rangle \varphi d x d t+\int_{\Omega}\left\langle\nu_{x, t}(\lambda), \eta(\lambda)\right\rangle \varphi(x, 0) d x
\end{gathered}
$$

(The weak formulation of boundary conditions) There exists a Radon measure $\vartheta_{s, t} \in \mathcal{M}_{b}\left(\partial \Omega \times \mathbb{R}^{+}\right)$such that $\forall \varphi \geq 0 \in \mathcal{C}_{c}^{1}\left(\bar{\Omega} \times \mathbb{R}^{+}\right), \quad \forall c \in \mathbb{R}$

$$
\begin{aligned}
\lim _{\delta \rightarrow 0} \mathcal{M}^{\eta^{\delta}}\left(\nu_{x, t}, \varphi\right) & -\int_{\partial \Omega \times \mathbb{R}^{+}} \operatorname{sgn}(b-c) \varphi(s, t) d \vartheta_{s, t} \\
& +\int_{\partial \Omega \times \mathbb{R}^{+}} F(c, x, t) \cdot n \operatorname{sgn}(b-c) \varphi d \sigma(x) d t \geq 0 .
\end{aligned}
$$


One emphasizes that the crucial point making this formulation equivalent to Szepessy's one (47), is the existence of the term $\operatorname{sgn}(b-c)$ in the weak formulation of both boundary terms (see Appendix B for the proof).

The remainder of the paper can be seen as an existence proof of a measure valued solution of equation (1) in the sense of definition (4.5). This existence follows by proving that $\bar{u}_{h}$, provided either by the scheme (36) or (45), are uniformly bounded in $L^{\infty}$ (section 6), weakly consistent with all entropy inequalities in the sense of definition (4.5), and finally consistent with the initial data (section 7). To this end, one needs to derive in the next section some preliminary properties of the particle scheme.

5. Basic properties of the upwind SPH scheme and entropy production. In connection with finite volume schemes ([2], [11], [22]), we have obtained in [4] a new interpretation of the hybrid particle scheme (36) in terms of one-dimensional finite difference schemes, as a sum of convex decomposition up to some additional terms (see (64) below). This interpretation allows us to get a suitable discrete maximum principle result yielding the derivation of the $L^{\infty}$ stability of $\bar{u}_{h}$.

For sake of clarity, when there is no ambiguity, we will use the notations for any function $G(u, x, t)$

$$
G_{k, k l}^{n}=G\left(u_{k}^{n}, x_{k l}, t^{n}\right), \quad G_{k, l}^{n}=G\left(u_{k}^{n}, x_{l}, t^{n}\right), \quad G_{\bar{u}_{h}}=G\left(\bar{u}_{h}, x, t\right) .
$$

5.1. Properties of the upwind particle scheme. Define the positive constant $\lambda_{k}^{n}$ by

$$
\lambda_{k}^{n}=\frac{\tau^{n}}{\Delta x_{k}^{n}}, \quad \Delta x_{k}^{n}=\frac{1}{\theta_{k}^{n}+\sum_{l \in K} \Gamma_{k l}^{n}}, \quad\left\{\begin{array}{l}
\Gamma_{k l}^{n}=2 w_{l}^{n} \chi_{k l}^{\kappa}\left\|\mathcal{A}_{k l}\right\| \\
\theta_{k}^{n}=\left\|\left(D_{\varepsilon, s}^{*} \chi^{\kappa}\right)_{k}^{n}\right\| .
\end{array}\right.
$$

Let also recall the numerical three point scheme which is purely one-dimensional, introduced in [4], defining the interaction between any pair $(k, l)$ of particles

$$
u_{k}^{n+1, l}=u_{k}^{n}-\lambda_{k}^{n}\left(g\left(n_{k l}, u_{k}^{n}, u_{l}^{n}\right)-F_{k, k l}^{n} . n_{k l}\right) \equiv W\left(\lambda_{k}^{n}, u_{k}^{n}, u_{l}^{n}\right) .
$$

Define in a similar way the interaction with boundary forces by

$$
u_{k}^{n+1, f}=u_{k}^{n}-\lambda_{k}^{n}\left(g\left(\tilde{n}_{k}, u_{k}^{n}, b_{k}^{n}\right)-F_{k, k}^{n} \cdot \tilde{n}_{k}\right) .
$$

So, to get the appropriate convex form of the scheme, one needs to find some positive constants $\alpha_{k}^{l, n}, \alpha_{k}^{k, n}$ with $\sum_{l \in K} \alpha_{k}^{l, n}+\alpha_{k}^{f, n}=1$ such that the scheme (ii) in (36) could be written as

$$
\tilde{u}_{k}^{n+1}=\sum_{l \in K} \alpha_{k}^{l, n} u_{k}^{n+1, l}+\alpha_{k}^{f, n} u_{k}^{n+1, f}+\tau^{n}\left(S_{k}^{n}-\mathcal{G}_{k}^{n}-\mathcal{B}_{k}^{n}\right) .
$$

Indeed, as in [4], a straightforward computation using the following choice

$$
\alpha_{k}^{f, n}=\frac{\theta_{k}^{n}}{\theta_{k}^{n}+\sum_{l \in K} \Gamma_{k l}^{n}}, \quad \alpha_{k}^{l, n}=\frac{\Gamma_{k l}^{n}}{\theta_{k}^{n}+\sum_{l \in K} \Gamma_{k l}^{n}},
$$


proves that (36) and (57) are equivalent if and only if

$$
\begin{aligned}
\mathcal{B}_{k}^{n} & =F_{k, k}^{n} \sum_{l \in K} w_{l}^{n} 2 \chi_{k l}^{\kappa} \mathcal{A}_{k l}^{n} \\
& =F_{k, k}^{n}\left(D_{\varepsilon, s}^{*} \chi^{\kappa}\right)_{k}^{n}+F_{k, k}^{n} \sum_{l \in K} w_{l}^{n}\left(2 \chi_{k l}^{\kappa}-\chi_{k}^{\kappa}-\chi_{l}^{\kappa}\right) \mathcal{A}_{k l}^{n} \\
& :=F_{k, k}^{n}\left(D_{\varepsilon, s}^{*} \chi^{\kappa}\right)_{k}^{n}+F_{k, k}^{n} \mathcal{R}\left(\chi^{\kappa}\right)_{k}^{n} \\
\mathcal{G}_{k}^{n} & =\sum_{l \in K} w_{l}^{n} 2 \chi_{k l}^{\kappa} F_{k, k l}^{n} \mathcal{A}_{k l}^{n} \\
& =\left(\partial_{\varepsilon, s}^{*} \chi^{\kappa} F_{\bar{u}_{h}}\right)_{k}^{n}+\sum_{l \in K} w_{l}^{n}\left(2 \chi_{k l}^{\kappa} F_{k, k l}^{n}-\chi_{k}^{\kappa} F_{k, k}^{n}-\chi_{l}^{\kappa} F_{k, l}^{n}\right) \mathcal{A}_{k l}^{n} \\
& :=\left(\partial_{\varepsilon, s}^{*} \chi^{\kappa} F_{\bar{u}_{h}}\right)_{k}^{n}+\mathcal{R}\left(\chi^{\kappa} F_{\bar{u}_{h}}\right)_{k}^{n} .
\end{aligned}
$$

The discrete partial derivative term $\partial_{\varepsilon, s}^{*}\left(\chi^{\kappa} F_{\bar{u}_{h}}\right)_{k}^{n}$ in the last equality denotes the adjoint operator associated with $\partial_{\varepsilon, s}\left(\chi^{\kappa} F_{\bar{u}_{h}}\right)_{k}^{n}$ according to (22). The latter one is nothing but the $\mathrm{SPH}$ approximation of the partial derivative

$$
\left(\sum_{i=1, d} \partial_{x^{i}}\left[\chi^{\kappa}(x) F\left(\bar{u}_{h}, x, t^{n}\right)\right]\right)_{\substack{x=x_{k}^{n}\\}},
$$

while the generic remainder $\mathcal{R}(.)_{k}^{n}$ is defined for any smooth function $g$ by

$$
\mathcal{R}(g)_{k}^{n}=\sum_{l \in K} w_{l}^{n}\left(2 g\left(x_{k l}^{n}\right)-g\left(x_{k}^{n}\right)-g\left(x_{l}^{n}\right)\right) \mathcal{A}_{k l}^{n} .
$$

To get the final form of the decomposition (57), we claim that

$$
\mathcal{B}_{k}^{n}+\mathcal{G}_{k}^{n}=\chi_{k}^{\kappa} \partial_{\varepsilon, s}^{*}\left(F_{\bar{u}_{h}}\right)_{k}^{n}+\mathcal{N}\left(\chi^{\kappa} F\right)_{k}^{n} \quad \text { with } \quad \lim _{\Delta(\varepsilon, h) \rightarrow 0} \mathcal{N}\left(\chi^{\kappa} F\right)_{k}^{n}=0 .
$$

Indeed, on the one hand, a direct computation gives for any smooth functions $f$ and $g$ that

$$
D_{\varepsilon, s}^{*}(f g)=f D_{\varepsilon, s}^{*} g+g D_{\varepsilon, s}^{*} f+\Xi(f, g)
$$

with

$$
\Xi(f, g)(x)=\sum_{l \in K} w_{l}\left(g(x)-g\left(x_{l}\right)\left(f(x)-f\left(x_{l}\right)\right) \nabla \zeta^{\varepsilon}\left(x-x_{l}\right)-2 g(x) f(x) D_{\varepsilon} 1(x) .\right.
$$

Thus, the estimate (12) and the bounds (14) imply that

$$
|\Xi(f, g)(x)| \leq C\left(\varepsilon+h / \varepsilon^{2}\right) .
$$

On the other hand, successive applications of Taylor expansion in $\left[x_{k}, x_{k l}\right]$ and $\left[x_{l}, x_{k l}\right]$ combined with the bounds (14) yield the following bounds

$$
\left\|\mathcal{R}\left(\chi^{\kappa}\right)_{k}^{n}\right\| \leq C \varepsilon \quad\left\|\mathcal{R}\left(\chi^{\kappa} F_{\bar{u}_{h}}\right)_{k}^{n}\right\| \leq C \varepsilon .
$$


Consequently, the combination of (61) applied to $\chi^{\kappa}$ and $F_{\bar{u}_{h}}$ and (62) ends the proof of the claim with

$$
\mathcal{N}\left(\chi^{\kappa} F\right)_{k}^{n}=\mathcal{R}\left(\chi^{\kappa} F\right)_{k}^{n}+F_{k, k}^{n} \cdot \mathcal{R}\left(\chi^{\kappa}\right)_{k}^{n}+\Xi\left(\chi^{\kappa}, F_{\bar{u}_{h}}\right) .
$$

Hence, the identity (57) reads

$$
\tilde{u}_{k}^{n+1}=\sum_{l \in K} \alpha_{k}^{l, n} u_{k}^{n+1, l}+\alpha_{k}^{f, n} u_{k}^{n+1, f}+\tau^{n}\left(S_{k}^{n}-\chi_{k}^{\kappa} \partial_{\varepsilon, s}^{*}\left(F_{\bar{u}_{h}}\right)_{k}^{n}+\mathcal{N}\left(\chi^{\kappa} F\right)_{k}^{n}\right) .
$$

To make appear the CFL condition (46), one first needs to rewrite the schemes (55) and (56) in terms of the incremental coefficient $C\left(n_{k l}, u_{k}^{n}, u_{l}^{n}\right)$ defined in (30). Secondly, on account of (14), one may show that there exist some constants $C, C_{-}$ and $C_{+}$, depending only on the velocity field $a$ and the kernel $\zeta$ such that

$$
\text { (i) } 0 \leq \theta_{k}^{n} \leq C / \varepsilon, \quad \text { (ii) } C_{-} \frac{\tau_{-}}{\varepsilon} \leq \lambda_{k}^{n} \leq C_{+} \frac{\tau_{+}}{\varepsilon} .
$$

5.2. Entropy production. In practice, we require that, for any convex entropy function $\eta$, there exists a numerical entropy flux $\hbar(n, u, v)$ satisfying similar requirements as the flux $g$ i.e.

$$
\text { (i) } \quad \hbar(n(x), u, u)=H(u, x, t) . n(x) \quad(\text { ii }) \quad \hbar(n, u, v)=-\hbar(-n, v, u) \text {, }
$$

where $H$ is an entropy flux associated with $(F, \eta)$, such that $\partial_{u} H^{i}=\eta^{\prime}(u) \partial_{u} F^{i}$. Moreover, such an entropy-entropy flux pair $(\eta, H)$ verifies a certain entropy inequality. Since we have an E-scheme, the incremental coefficient $C(n, u, v)$ is positive. Therefore, by using the three point scheme (55), it follows from Proposition 3.3 in [2] (the $(x, t)$ dependence is omitted below) that for the entropy-entropy flux pair $\left(\eta=u^{2}, H\right)$,

$$
\eta(W(\lambda, u, v))-\eta(u)+\lambda[\hbar(n, u, v)-H(u) n] \leq-\frac{\beta}{2}|u-v|^{2}(C(n, u, v))^{2} \lambda^{2},
$$

provided the CFL condition $\lambda Q \leq 1-\beta$ is satisfied. Note however that (66) is valid for any entropy-entropy flux pair if we take $\beta=0$, in which case, we have

$$
\hbar(n, u, v)-H(u) . n \leq \eta^{\prime}(u)(g(n, u, v)-F(u) . n) .
$$

Moreover, in view of treating the boundary contribution, a straightforward calculations proves that for $\eta=\eta_{c}=|u-c|$ the inequality (67) is equivalent to

$$
\operatorname{sgn}(v-c)(g(u, v)-F(c)) \leq \hbar_{c}(u, v) \leq \operatorname{sgn}(u-c)(g(u, v)-F(c)) .
$$

Let us now turn to the derivation of the entropy dissipation corresponding to our hybrid particle scheme.

In the interior domain: The inequality (66) reads

$$
\begin{aligned}
\eta\left(u_{k}^{n+1, l}\right)-\eta\left(u_{k}^{n}\right)+\lambda_{k}^{n}( & \left.\hbar\left(n_{k l}, u_{k}^{n}, u_{l}^{n}\right)-H_{k, k l}^{n} . n_{k l}\right) \\
& \leq-\frac{\beta}{2}\left|u_{k}-u_{l}\right|^{2}\left(C\left(n_{k l}, u_{k}^{n}, u_{l}^{n}\right)\right)^{2}\left(\lambda_{k}^{n}\right)^{2} .
\end{aligned}
$$


By adding and substituting appropriate terms, this last inequality reads

$$
\begin{aligned}
& \eta\left(u_{k}^{n+1, l}\right)-\eta\left(u_{k}^{n}\right)+\frac{\lambda_{k}^{n}}{2}\left(H_{k, k}^{n}+H_{l, l}^{n}\right) n_{k l}-\lambda_{k}^{n} H_{k, k l}^{n} \cdot n_{k l} \\
& \quad \leq-\frac{\lambda_{k}^{n}}{2}\left(2 \hbar\left(n_{k l}, u_{k}^{n}, u_{l}^{n}\right)-\left(H_{k, k l}^{n}+H_{l, l k}^{n}\right) n_{k l}\right) \\
& \quad-\frac{\lambda_{k}^{n}}{2}\left(H_{k, k l}^{n}+H_{l, l k}^{n}-H_{k, k}^{n}-H_{l, l}^{n}\right) . n_{k l}-\frac{\beta}{2}\left|u_{k}-u_{l}\right|^{2}\left(C\left(n_{k l}, u_{k}^{n}, u_{l}^{n}\right)\right)^{2}\left(\lambda_{k}^{n}\right)^{2} .
\end{aligned}
$$

Multiplying this last inequality by $\alpha_{k}^{l, n}$, summing it over $(l \in K)$ and using (54) and (58), we then get

$$
\begin{aligned}
& \sum_{l \in K} \alpha_{k}^{l, n}\left(\eta\left(u_{k}^{n+1, l}\right)-\right.\left.\eta\left(u_{k}^{n}\right)\right)+\tau^{n} D_{\varepsilon, s}^{*}\left(\chi^{\kappa} H_{\bar{u}_{h}}\right)_{k}^{n} \\
&-\tau^{n} \partial_{\varepsilon, s}^{*}\left(\chi^{\kappa} H_{\bar{u}_{h}}\right)_{k}^{n} \leq \mathcal{R}\left(\chi^{\kappa} H\right)_{k}^{n}-\sum_{i=1,3}\left(\mu_{h, \varepsilon}^{i}\right)_{k}^{n} \\
& \quad-\sum_{l \in K} \tau^{n} w_{l}^{n} \lambda_{k}^{n}\left|u_{k}^{n}-u_{l}^{n}\right|^{2}\left(C\left(n_{k l}, u_{k}^{n}, u_{l}^{n}\right)\right)^{2}\left\|\mathcal{A}_{k l}\right\|,
\end{aligned}
$$

where the remainder $\mathcal{R}\left(\chi^{\kappa} H\right)_{k}^{n}$ is defined according to (60) and the above measure terms are given by

$\left(\mu_{h, \varepsilon}^{1}\right)_{k}^{n}=\tau^{n} \sum_{l \in K} w_{l}^{n} \chi_{k l}^{\kappa}\left[2 \hbar\left(n_{k l}, u_{k}^{n}, u_{l}^{n}\right)-\left(H_{k, k l}^{n}-H_{l, l k}^{n}\right) . n_{k l}\right]\left\|\mathcal{A}_{k l}\right\|$,

$\left.\left(\mu_{h, \varepsilon}^{2}\right)_{k}^{n}=\tau^{n} \sum_{l \in K} w_{l}^{n} \chi_{k l}^{\kappa}\left[\left(H_{k, k l}^{n}+H_{l, l k}^{n}\right)-\left(H_{k, k}^{n}+H_{l, l}^{n}\right)\right)\right] \mathcal{A}_{k l}$,

$\left(\mu_{h, \varepsilon}^{3}\right)_{k}^{n}=\tau^{n} \sum_{l \in K} w_{l}^{n}\left(\left(\chi_{k l}^{\kappa}-\chi_{k}^{\kappa}\right) H_{k, k}^{n}+\left(\chi_{k l}^{\kappa}-\chi_{l}^{\kappa}\right) H_{l, l}^{n}\right) \mathcal{A}_{k l}$

The boundary contribution: In a similar way, by using the three point scheme (56) and the entropy inequality (66), one gets

$$
\begin{aligned}
& \alpha_{k}^{f, n}\left[\eta\left(u_{k}^{n+1, f}\right)-\eta\left(u_{k}^{n}\right)\right]+\tau^{n} \theta_{k}^{n} \hbar\left(\tilde{n}_{k}, u_{k}^{n}, b_{l}^{n}\right)-\tau^{n} H_{k, k}^{n} . D_{\varepsilon, s}^{*}\left(\chi^{\kappa}\right)_{k}^{n} \\
& \quad \leq \tau^{n} H_{k, k}^{n} \cdot \mathcal{R}\left(\chi^{\kappa}\right)_{k}^{n}-\frac{\beta}{2} \tau^{n} \theta_{k}^{n}\left|u_{k}-b_{l}\right|^{2}\left(C\left(\tilde{n}_{k}, u_{k}, b_{k}\right)\right)^{2}\left(\lambda_{k}^{n}\right) .
\end{aligned}
$$

The resulting interaction: Combining the two inequalities (70) and (71), and using the identity (61), it yields

$$
\begin{aligned}
& \sum_{l \in K} \alpha_{k}^{l, n}\left(\eta\left(u_{k}^{n+1, l}\right)+\right. \alpha_{k}^{f, n}\left(\eta\left(u_{k}^{n+1, f}\right)-\eta\left(u_{k}^{n}\right)\right. \\
&+D_{\varepsilon, s}^{*}\left(\chi^{\kappa} H_{\bar{u}_{h}}\right)_{k}^{n}-\tau^{n} \chi_{k}^{\kappa}\left(\partial_{\varepsilon, s}^{*} H_{\bar{u}_{h}}\right)_{k}^{n}+\tau^{n} \theta_{k}^{n} \hbar\left(\tilde{n}_{k}, u_{k}^{n}, b_{k}^{n}\right) \\
& \leq \tau^{n} \mathcal{N}\left(\chi^{\kappa}, H\right)_{k}^{n}-\sum_{i=1,3}\left(\mu_{h, \varepsilon}^{i}\right)_{k}^{n}-\beta \Delta_{k}^{n}(u),
\end{aligned}
$$

where the remainder $\mathcal{N}\left(\chi^{\kappa}, H\right)_{k}^{n}$ is defined according to (63) and

$$
\begin{aligned}
\Delta_{k}^{n}(u)=2 \sum_{l \in K} \tau^{n} w_{l}^{n} \lambda_{k}^{n}\left|u_{k}^{n}-u_{l}^{n}\right|^{2}\left(C\left(n_{k l}, u_{k}^{n}, u_{l}^{n}\right)\right)^{2}\left\|\mathcal{A}_{k l}\right\| \\
+2 \tau^{n} \theta_{k}^{n} \lambda_{k}^{n}\left|u_{k}^{n}-b_{k}^{n}\right|^{2}\left(C\left(\tilde{n}_{k}, u_{k}, b_{k}\right)\right)^{2} .
\end{aligned}
$$


So, by using successively the convexity inequaliy of $\eta$, the new form (64), the inequality $\eta(u+v) \leq \eta(u)+v \eta^{\prime}(u+v)$ together with Jensen's inequality, we then obtain the final form of the entropy inequality

$$
\begin{gathered}
\eta\left(\tilde{u}_{k}^{n+1}\right)-\eta\left(u_{k}^{n}\right)+D_{\varepsilon, s}^{*}\left(\chi^{\kappa} H_{\bar{u}_{h}}\right)_{k}^{n}+\tau^{n} \theta_{k}^{n} \hbar\left(\tilde{n}_{k}, u_{k}^{n}, b_{k}^{n}\right) \\
-\tau^{n}\left(\chi_{k}^{\kappa}\left(\partial_{\varepsilon, s} H_{\bar{u}_{h}}\right)_{k}^{n}-\eta^{\prime}\left(u_{k}^{n}\right)\left(S_{k}^{n}-\chi_{k}^{\kappa} \partial_{\varepsilon, s}\left(F_{\bar{u}_{h}}\right)_{k}^{n}\right)\right) \\
\leq-\sum_{i=1,5}\left(\mu_{h, \varepsilon}^{i}\right)_{k}^{n}-\beta \Delta_{k}^{n}(u)
\end{gathered}
$$

where the additional measure terms are defined by

$\left(\mu_{h, \varepsilon}^{4}\right)_{k}^{n}=\tau^{n}\left(\eta^{\prime}\left(\tilde{u}_{k}^{n+1}\right)-\eta^{\prime}\left(u_{k}^{n}\right)\right)\left(S_{k}^{n}-\chi_{k}^{\kappa} D_{\varepsilon, s}^{x}\left(F_{\bar{u}_{h}}\right)_{k}^{n}\right)$

$\left(\mu_{h, \varepsilon}^{5}\right)_{k}^{n}=\tau^{n}\left(\mathcal{N}\left(\chi^{\kappa}, H\right)_{k}^{n}-\eta^{\prime}\left(\tilde{u}_{k}^{n+1}\right) \mathcal{N}\left(\chi^{\kappa}, F\right)_{k}^{n}\right)$.

6. $L^{\infty}$ Stability and weak BV estimate. Let us denote by the sequence $v_{k}^{n+1}$ the convex part in the scheme (64) i.e.

$$
v_{k}^{n+1}=\sum_{l \in K} \alpha_{k}^{l, n} u_{k}^{n+1, l}+\alpha_{k}^{f, n} u_{k}^{n+1, f}
$$

Proposition 6.1. Assume that $\left(u_{0}, b\right) \in L^{\infty}(\Omega) \times L^{\infty}\left(\partial \Omega \times \mathbb{R}_{+}\right)$and $\bar{u}_{h}(x, t)$ is computed by the scheme (36-38) with E-fluxes, then for any $T>0$, provided the time step $\tau^{n}$ satisfies the CFL condition (46), we successively have

$$
\begin{gathered}
\text { (i) } \min _{\substack{i \in K \\
j \in N_{e}}}\left(u_{i}^{n}, b_{j}^{n}\right) \leq v_{k}^{n+1} \leq \max _{\substack{i \in K \\
j \in N_{e}}}\left(u_{i}^{n}, b_{j}^{n}\right) \\
\left\|\bar{u}_{h}(., t)\right\|_{\infty} \leq K_{\infty}:=C\left(T,\left\|u_{0}\right\|_{\infty},\|b\|_{\infty}\right) \\
\text { (ii) } \sum_{\substack{k \in K \\
n ; t^{n} \leq T}} w_{k}^{n} \Delta_{k}^{n}(u) \leq C_{\beta} \equiv \frac{1}{\beta} \times\left(\left\|u_{0}\right\|_{L^{2}}^{2}+C\left(T,\left\|u_{0}\right\|_{\infty},\|b\|_{\infty}\right)\right),
\end{gathered}
$$

where the local dissipation term $\Delta_{k}^{n}(u)$ is given by (73).

Proof of proposition 6.1.

- $L^{\infty}$ stability: Rewriting the schemes (55) and (56) by means of the incremental coefficient $C$ given by (30), then the CFL condition (46) implies that $u_{k}^{n+1, l} \in I\left(u_{k}^{n}, u_{l}^{n}\right)$ and $u_{k}^{n+1, f} \in I\left(u_{k}^{n}, b_{l}^{n}\right)$ with

$$
I(f, g):=\{w ; w=\theta f+(1-\theta) g, \theta \in[0,1]\} .
$$

Therefore, the inequality $(i)$ follows directly. To prove the $L^{\infty}$ stability, consider the increasing function $v \longrightarrow G(v, T)$ defined by

$$
G(v, T)=\max _{\substack{|u| \leq v \\ x \in \Omega, t \leq T}}\left(\left\|\partial_{x} F(u, x, t)\right\|+\|F(u, x, t)\|+\|S(u, x, t)\|\right),
$$

and start with the new form of the scheme (64). Thus, using successively the estimates (13) and (63), we find that

$$
\left|u_{k}^{n+1}\right| \leq \frac{w_{k}^{n}}{w_{k}^{n+1}} \times\left(\left\|u^{n}\right\|_{\infty}+\|b\|_{\infty}+\tau^{n} G\left(\left\|u^{n}\right\|_{\infty}, T\right) C\left(1+\varepsilon+\frac{h}{\varepsilon^{2}}\right)\right) .
$$


Take $C_{\mathbf{a}}=\|\operatorname{div} \mathbf{a}\|_{\infty} \exp \left(\|\operatorname{div} \mathbf{a}\|_{\infty} T\right)$ then, the identity (38) yields $w_{k}^{n} / w_{k}^{n+1} \leq(1+$ $\left.C_{\mathbf{a}} \tau^{n}\right)$ and consequently,

$$
\left|u_{k}^{n+1}\right| \leq\left(1+C_{\mathbf{a}} \tau^{n}\right) \times\left(\left\|u^{n}\right\|_{\infty}+\|b\|_{\infty}+\tau^{n} G\left(\left\|u^{n}\right\|_{\infty}, T\right) C\left(1+\varepsilon+\frac{h}{\varepsilon^{2}}\right)\right) .
$$

Next, as in [4], define the sequence $v^{n}$ by

$$
\left\{\begin{array}{l}
v^{0}=\left\|u^{0}\right\|_{\infty}, \\
v^{n+1}+\|b\|_{\infty}=\left(1+C_{\mathbf{a}} \tau^{n}\right)\left(v^{n}+\|b\|_{\infty}+\tau^{n} G\left(v^{n}, T\right) C\left(1+\varepsilon+\frac{h}{\varepsilon^{2}}\right)\right) .
\end{array}\right.
$$

By construction, $\left\|u^{n}\right\|_{\infty} \leq v^{n}$ and from the inequality (76) we infer that

$$
\begin{aligned}
\frac{v^{n+1}-v^{n}}{\tau^{n}} & \leq C\left(C_{\mathbf{a}}, T\right)\left(v^{n}+\|b\|_{\infty}\right)+C\left(1+\varepsilon+\frac{h}{\varepsilon^{2}}\right) G\left(v^{n}, T\right) \\
& \equiv \phi\left(v^{n}\right) .
\end{aligned}
$$

Let $\Phi(v):=\int_{v^{0}}^{v} \frac{1}{\phi(x)} d x ; \Phi$ is a smooth and increasing function, its converse function also. We easily get that : $\Phi\left(v^{n+1}\right) \leq\left(t^{n+1}-t^{0}\right)+\Phi\left(v^{0}\right)$ and the following $L^{\infty}$ estimate holds

$$
\left\|u^{n}\right\|_{\infty} \leq \Phi^{-1}\left(\left(t^{n}-t^{0}\right)+\Phi\left(v^{0}\right)\right) .
$$

Weak $B V$ estimate (iii): Take the inequality (74) with $\beta \neq 0$ and $\eta(u)=u^{2} / 2$, then the multiplication by $w_{k}^{n}$ and the summation over $k \in K$ yield

$$
\begin{gathered}
\sum_{k \in K} w_{k}^{n}\left[\eta\left(\tilde{u}_{k}^{n+1}\right)-\eta\left(u_{k}^{n}\right)+D_{\varepsilon, s}^{*}\left(\chi^{\kappa} H_{\bar{u}_{h}}\right)_{k}^{n}+\tau^{n} \theta_{k}^{n} \hbar\left(\tilde{n}_{k}, u_{k}^{n}, b_{k}^{n}\right)\right] \\
-\tau^{n} \sum_{k \in K} w_{k}^{n}\left(\chi_{k}^{\kappa}\left(\partial_{\varepsilon, s} H_{\bar{u}_{h}}\right)_{k}^{n}-\eta^{\prime}\left(u_{k}^{n}\right)\left(S_{k}^{n}-\chi_{k}^{\kappa} \partial_{\varepsilon, s}\left(F_{\bar{u}_{h}}\right)_{k}^{n}\right)\right) \\
\leq-\sum_{i=1,5} \sum_{k \in K} w_{k}^{n}\left(\mu_{h}^{i}\right)_{k}^{n}-\beta \sum_{k \in K} w_{k}^{n} \Delta_{k}^{n}(u) .
\end{gathered}
$$

Notice that, switching the indices $k$ and $l$ and using that $\mathcal{A}_{k l}=-\mathcal{A}_{l k}$ together with the identity $\hbar\left(n_{k l}, u_{k}^{n}, u_{l}^{n}\right)=-\hbar\left(n_{l k}, u_{l}^{n}, u_{k}^{n}\right)$, one deduces

$$
\sum_{k \in K} w_{k}^{n} D_{\varepsilon, s}^{*}\left(\chi^{\kappa} H_{\bar{u}_{h}}\right)_{k}^{n}=0=\sum_{i=1}^{3}\left|\sum_{k \in K} w_{k}^{n}\left(\nu_{h}^{i}\right)_{k}^{n}\right| .
$$

Moreover, adding and substituting the term $\sum_{k \in K} w_{k}^{n+1} \eta\left(u_{k}^{n+1}\right)$ and summing up the inequality (77) over $t^{n} \leq T=t^{N}$, we find that

$$
\begin{aligned}
& \sum_{k \in K}\left(w_{k}^{N}\left|u_{k}^{N}\right|^{2}-w_{k}^{0}\left|u_{k}^{0}\right|^{2}\right)+\beta \sum_{\substack{k \in K \\
n ; t^{n} \leq T}} w_{k}^{n} \Delta_{k}^{n}(u)+\sum_{i=4,5} \sum_{k \in K} w_{k}^{n}\left(\mu_{h}^{i}\right)_{k}^{n} \\
& \leq \sum_{\substack{k \in K \\
n ; t^{n} \leq T}} \tau^{n} w_{k}^{n}\left(\chi_{k}^{k}\left(\partial_{\varepsilon, s}^{*} H_{\bar{u}_{h}}\right)_{k}^{n}-u_{k}^{n}\left(S_{k}^{n}-\chi_{\kappa}^{k} \partial_{\varepsilon, s}^{*}\left(F_{\bar{u}_{h}}\right)_{k}^{n}\right)\right) \\
& \quad+\sum_{\substack{k \in K \\
n ; t^{n} \leq T}} \tau^{n} w_{k}^{n} \theta_{k}^{n} \hbar\left(\tilde{n}_{k}, u_{k}^{n}, b_{k}^{n}\right)+\sum_{\substack{k \in K \\
n ; t^{n} \leq T}}\left(w_{k}^{n+1}\left|u_{k}^{n+1}\right|^{2}-w_{k}^{n}\left|\tilde{u}_{k}^{n+1}\right|^{2}\right) \\
& \equiv R^{2}+R^{3}+R^{4} .
\end{aligned}
$$


Thus, straightforward computations using successively the identities (36) (iii) and (38), the Lipschitz continuity of the numerical flux $\hbar(n, u, v)$, the fact that $\operatorname{supp}(\theta(x)) \subset \partial \Omega \times\left[0, \kappa^{\prime}\right]$ together with the estimates (11-13) and (65) (ii) imply the following estimates

$$
\left|R^{2}\right|+\left|R^{3}\right| \leq C\left(T, \varepsilon, h / \varepsilon^{2}, \kappa^{\prime} / \varepsilon,\left\|u^{0}\right\|_{\infty},\|b\|_{\infty}\right), \quad\left|R^{4}\right| \leq C\left(T, \frac{\kappa^{\prime}}{\varepsilon},\left\|\bar{u}_{h}\right\|_{L^{2}}\right) .
$$

On the other hand, since $\eta(u)=u^{2} / 2$, we have

$$
\eta^{\prime}\left(\tilde{u}_{k}^{n+1}\right)-\eta^{\prime}\left(u_{k}^{n}\right)=\tilde{u}_{k}^{n+1}-u_{k}^{n},
$$

therefore, in view of (36) (ii), the combination of the bounds (14) and (62) with the CFL condition (46) gives

$$
\sum_{i=4,5}\left|\sum_{k \in K} w_{k}^{n}\left(\mu_{h}^{i}\right)_{k}^{n}\right| \leq C\left(T, \varepsilon,\left\|u^{0}\right\|_{\infty},\|b\|_{\infty}\right) .
$$

By taking the results (79) and (80) into account, the inequality (78) yields the weak estimate (75) (iii).

7. Proof of Theorem 4.1. The proof of this theorem will be split into three steps. In the first step, we derive the global weak entropy formulation of the scheme (36), while the last two steps are devoted to the existence proof of a measure-valued solution in the sense of the definition (4.5).

First step: Weak entropy form of the scheme. In connection with the discrete scalar product used in (22), we are going to use the following notation whenever a time integration is added

$$
(g, f)_{h, T}=\sum_{\substack{\left\{n, t^{n} \leq T\right\} \\ k \in \bar{K}}} w_{k}^{n} g_{k}^{n} f_{k}^{n}=\int_{\mathcal{Q}_{T}} g(x, t) f(x, t) d x d t+\mathcal{E}^{h, T}(g f),
$$

where $\mathcal{Q}_{T}=\Omega \times[0, T]$ and $\mathcal{E}^{h, T}(g f)$ denotes the resulting quadrature error. Thus, we have

Proposition 7.1. For all nonnegative test function $\varphi \in \mathcal{C}_{c}^{1}\left(\bar{\Omega} \times \mathbb{R}_{+}\right)$, the approximate solutions $\bar{u}_{h}$ defined by (23) and computed by the scheme (36)-(38) satisfy

$$
\begin{aligned}
\mathcal{M}^{\eta}\left(\delta_{\bar{u}_{h}(x, t)}, \chi^{\kappa}, \varphi\right)-\Gamma(\varphi)_{h, T}^{\kappa^{\prime}} & \geq \sum_{i=1,7}<\mu_{h, \varepsilon}^{i}, \varphi>_{\mathcal{Q}_{T}}+\sum_{m=1,4} \mathcal{R}_{m}^{h, \varepsilon} \\
& \equiv<\mu_{h, \varepsilon}, \varphi>_{\mathcal{Q}_{T}},
\end{aligned}
$$

where

$$
\begin{aligned}
& \mathcal{M}^{\eta}\left(\delta_{\bar{u}_{h}(x, t)}, \mathbf{a}, \chi^{\kappa}, \varphi\right):= \int_{\Omega \times \mathbb{R}_{+}}\left[\eta\left(\bar{u}_{h}\right) \mathcal{L}_{\mathbf{a}}^{*}(\varphi)+\chi^{\kappa} \hbar\left(\bar{u}_{h}, x, t\right) \cdot D_{\varepsilon, s} \varphi\right] d x d t \\
&+\int_{\Omega \times \mathbb{R}_{+}}\left[\left(\eta\left(\bar{u}_{h}\right)-\eta^{\prime}\left(\bar{u}_{h}\right) \bar{u}_{h}\right) \operatorname{div}(\mathbf{a}(x, t)] \varphi d x d t\right. \\
&+\int_{\Omega \times \mathbb{R}_{+}} \chi^{\kappa}\left(\partial_{\varepsilon, s}^{*} H_{\bar{u}_{h}}-\eta^{\prime}\left(\bar{u}_{h}\right) \partial_{\varepsilon, s}^{*} F_{\bar{u}_{h}}\right) \varphi d x d t \\
&+\int_{\Omega \times \mathbb{R}_{+}} \eta^{\prime}\left(\bar{u}_{h}\right) S_{\bar{u}_{h}} \varphi d x d t+\int_{\Omega} \eta\left(\bar{u}_{h}(x, 0)\right) \varphi(x, 0) d x \\
& \Gamma(\varphi)_{h, T}^{\kappa^{\prime}}=\sum_{\substack{\left\{n, t^{n} \leq T\right\} \\
k \in K}} \tau^{n} w_{k}^{n} \theta_{k}^{n} \hbar\left(\tilde{n}_{k}, u_{k}^{n}, b_{k}^{n}\right) \varphi_{k}^{n},
\end{aligned}
$$


while the right hand side $\left\langle\mu_{h, \varepsilon}, \varphi>_{\mathcal{Q}_{T}}\right.$ will be made precise below.

Proof of proposition 7.1. As in [4], start with the inequality (74) for all convex entropy $\eta$ (i.e. $\beta=0$ ), multiply it by $\varphi_{k}^{n} w_{k}^{n}$ and take the double sum over $\left\{n, t^{n} \leq T\right\}$ and $k \in K$, on the one hand. On the other hand, making an integration by parts and using that $\mathcal{A}_{k l}=-\mathcal{A}_{l k}$ together with the notation (81), one gets

$$
\begin{aligned}
& -\sum_{\substack{\left\{n, t^{n} \leq T\right\} \\
k \in \bar{K}}} \varphi_{k}^{n} w_{k}^{n}\left(\eta\left(\tilde{u}_{k}^{n+1}\right)-\eta\left(u_{k}^{n}\right)\right)+\left(\hbar\left(\bar{u}_{h}, x, t\right), \chi^{\kappa} D_{\varepsilon, s} \varphi\right)_{h, T} \\
& +\left(\varphi, \chi^{\kappa}\left(\partial_{\varepsilon, s}^{*}\left(H_{\bar{u}_{h}}\right)_{k}^{n}-\eta^{\prime}\left(\bar{u}_{h}\right)\left(\chi_{k}^{\kappa} \partial_{\varepsilon, s}^{*}\left(F_{\bar{u}_{h}}\right)_{k}^{n}-S_{\bar{u}_{h}}\right)\right)_{h, T}\right. \\
& -\Gamma(\varphi)_{h, T}^{\kappa^{\prime}} \leq \sum_{i=1}^{5}<\mu_{h, \varepsilon}^{i}, \varphi>_{\mathcal{Q}_{T}},
\end{aligned}
$$

where the right hand side is given by

$$
\begin{aligned}
<\mu_{h, \varepsilon}^{1}, \varphi>_{\mathcal{Q}_{T}}=-\sum_{\substack{\left\{n, t^{n} \leq T\right\} \\
(k, l) \in K^{2}}} \tau^{n} w_{k}^{n} w_{l}^{n} \chi_{k l}^{\kappa}\left(\varphi_{k}^{n}-\varphi_{l}^{n}\right)\left[\hbar\left(n_{k l}, u_{k}^{n}, u_{l}^{n}\right)-H_{k l}^{n} \cdot n_{k l}\right]\left\|\mathcal{A}_{k l}\right\| \\
<\mu_{h, \varepsilon}^{2}, \varphi>_{\mathcal{Q}_{T}}=-\sum_{\substack{\left\{n, t^{n} \leq T\right\} \\
(k, l) \in K^{2}}} \tau^{n} w_{k}^{n} w_{l}^{n} \chi_{k l}^{\kappa}\left(\varphi_{k}^{n}-\varphi_{l}^{n}\right)\left(H_{l}^{n}-H_{l, l k}^{n}\right) \cdot \mathcal{A}_{k l} \\
<\mu_{h, \varepsilon}^{3}, \varphi>_{\mathcal{Q}_{T}}=\sum_{\substack{\left\{n, t^{n} \leq T\right\} \\
(k, l) \in K^{2}}} \tau^{n} w_{k}^{n} \tau^{n} w_{k}^{n} w_{l}^{n}\left(\varphi_{k}^{n}-\varphi_{l}^{n}\right)\left(\chi_{k l}^{\kappa}-\chi_{k}^{\kappa}\right) H_{k}^{n} \cdot \mathcal{A}_{k l} \\
<\mu_{h, \varepsilon}^{4}, \varphi>_{\mathcal{Q}_{T}}=-\sum_{\substack{\left\{n, t^{n} \leq T\right\} \\
k \in K}} \tau^{n} w_{k}^{n} \varphi_{k}^{n}\left(\eta^{\prime}\left(\tilde{u}_{k}^{n+1}\right)-\eta^{\prime}\left(u_{k}^{n}\right)\right)\left[-\chi_{k}^{\kappa} \partial_{\varepsilon, s}^{*}\left(F_{\bar{u}_{h}}\right)_{k}^{n}+S_{k}^{n}\right] \\
<\mu_{h, \varepsilon}^{5}, \varphi>_{\mathcal{Q}_{T}}=-\sum_{\substack{\left\{n, t^{n} \leq T\right\} \\
k \in K}} \tau^{n} w_{k}^{n} \varphi_{k}^{n}\left(\mathcal{N}\left(\chi^{\kappa}, H\right)_{k}^{n}-\eta^{\prime}\left(\tilde{u}_{k}^{n+1}\right) \mathcal{N}\left(\chi^{\kappa}, F\right)_{k}^{n}\right) .
\end{aligned}
$$

Let us now denote by $\mathcal{D}$ the first term in the left hand side of $(83)$; then, one establishes with similar arguments as before (see [4] for the detailed proof) that

$$
\begin{aligned}
\mathcal{D} \geq & \left(\eta\left(\bar{u}_{h}\right), \mathcal{L}_{\mathbf{a}}^{*} \varphi\right)_{h, T}+\left(\eta\left(\bar{u}_{h}\right), \varphi\right)_{h} \\
& +\left(\left(\eta\left(\bar{u}_{h}\right)-\eta^{\prime}\left(\bar{u}_{h}\right) \bar{u}_{h}\right) \operatorname{diva}, \varphi\right)_{h, T}+<\mu_{h, \varepsilon}^{6}+\mu_{h, \varepsilon}^{7}, \varphi>_{\mathcal{Q}_{T}},
\end{aligned}
$$

where the above measure terms are defined as follows

$$
\begin{aligned}
& <\mu_{h, \varepsilon}^{6}, \varphi>_{\mathcal{Q}_{T}}=-\sum_{\substack{n, t^{n} \leq T \\
k \in K}}\left[\omega_{k}^{n+1} \eta\left(u_{k}^{n+1}\right)-\omega_{k}^{n} \eta\left(u_{k}^{n}\right)\right]\left(\varphi_{k}^{n+1}-\varphi_{k}^{n}\right) \\
& <\mu_{h, \varepsilon}^{7}, \varphi>_{\mathcal{Q}_{T}}= \\
& =\sum_{\substack{n, t^{n} \leq T \\
k \in K}} \varphi_{k}^{n}\left[\left(\eta^{\prime}\left(u_{k}^{n+1}\right) \tilde{u}_{k}^{n+1}-\eta\left(\tilde{u}_{k}^{n+1}\right)\right)-\left(\eta^{\prime}\left(u_{k}^{n}\right) u_{k}^{n}-\eta\left(u_{k}^{n}\right)\right)\right]\left(\omega_{k}^{n+1}-\omega_{k}^{n}\right) .
\end{aligned}
$$


Thus, the combination of the inequalities (84) and (83) yields

$$
\begin{aligned}
& \left(\eta\left(\bar{u}_{h}\right), \mathcal{L}_{\mathbf{a}}^{*} \varphi\right)_{h, T}+\left(\eta\left(\bar{u}_{h}\right)-\eta^{\prime}\left(\bar{u}_{h}\right) \bar{u}_{h} \operatorname{diva}, \varphi\right)_{h, T} \\
& +\left(H_{\bar{u}_{h}}, \chi^{\kappa} D_{\varepsilon, s} \varphi\right)_{h, T}+\left(\varphi, \chi^{\kappa} \partial_{\varepsilon, s}^{*}\left(H_{\bar{u}_{h}}\right)-\eta^{\prime}\left(\bar{u}_{h}\right)\left(\chi^{\kappa} \partial_{\varepsilon, s}^{*}\left(F_{\bar{u}_{h}}\right)-S_{\bar{u}_{h}}\right)\right)_{h, T} \\
& \quad-\left(\mu_{h, \varepsilon}^{0}, \varphi\right)_{h, T}^{\kappa^{\prime}}+\left(\eta\left(\bar{u}_{h}\right), \varphi\right)_{h} \leq \sum_{i=1}^{7}<\mu_{h, \varepsilon}^{i}, \varphi>_{\mathcal{Q}_{T}} .
\end{aligned}
$$

Finally, the desired inequality (82) follows by using the decomposition in (81) and denoting by $\mathcal{R}_{i}^{h, \varepsilon}$ for $i \in\{1, . .4\}$ the corresponding quadrature error terms on $\mathcal{Q}_{T}$ and on $\Omega$ for the initial data

$$
\begin{aligned}
& \mathcal{R}_{1}^{h, \varepsilon}=\mathcal{E}^{h, T}\left(\eta\left(\bar{u}_{h}\right) \mathcal{L}_{\mathbf{a}}^{*} \varphi+\left(\eta\left(\bar{u}_{h}\right)-\eta^{\prime}\left(\bar{u}_{h}\right) \bar{u}_{h}\right) \varphi \operatorname{diva}\right), \quad \mathcal{R}_{2}^{h, \varepsilon}=\mathcal{E}^{h, T}\left(H_{\bar{u}_{h}} \chi^{\kappa} D_{\varepsilon, s} \varphi\right) \\
& \mathcal{R}_{3}^{h, \varepsilon}=\mathcal{E}^{h, T}\left(\varphi\left(\chi^{\kappa} \partial_{\varepsilon, s}^{*} H_{\bar{u}_{h}}-\eta^{\prime}\left(\bar{u}_{h}\right)\left(\chi^{\kappa} \partial_{\varepsilon, s}^{*} F_{\bar{u}_{h}}-S_{\bar{u}_{h}}\right)\right)\right), \quad \mathcal{R}_{4}^{h, \varepsilon}=\mathcal{E}^{h}\left(\eta\left(\bar{u}_{h}\right) \varphi\right) .
\end{aligned}
$$

Second step: Derivation of (47). On account of the $L^{\infty}$ stability result of $\bar{u}_{h}$ (75) (ii) and following [43] and [13], one can extract a subsequence $\left\{\bar{u}_{h_{j}}\right\}$ with an associated Young measure-valued mapping $\nu_{(\cdot)}: \Omega \times \mathbb{R}_{+} \rightarrow \operatorname{Prob}\left(\left[-K_{\infty}, K_{\infty}\right]\right.$ ) (see (75) (ii) for the value of the constant $K_{\infty}$ ), such that

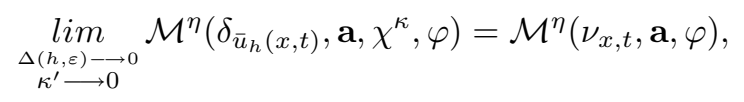

where $\mathcal{M}^{\eta}\left(\nu_{x, t}, \mathbf{a}, \varphi\right)$ is given by

$$
\begin{aligned}
\mathcal{M}^{\eta}\left(\nu_{x, t}, \mathbf{a}, \varphi\right) & :=\int_{\Omega \times \mathbb{R}_{+}}\left\{\left\langle\nu_{x, t}(\lambda), \eta(\lambda)\right\rangle \mathcal{L}_{\mathbf{a}}^{*}(\varphi)+\left\langle\nu_{x, t}(\lambda), \hbar(\lambda, x, t)\right\rangle \nabla_{x} \varphi d x d t\right. \\
& +\int_{\Omega \times \mathbb{R}_{+}}\left\langle\nu_{x, t}(\lambda),\left(\eta(\lambda)-\eta^{\prime}(\lambda) \lambda\right)\right\rangle \operatorname{div}(\mathbf{a}(x, t)) \varphi d x d t \\
& +\int_{\Omega \times \mathbb{R}_{+}}\left\langle\nu_{x, t}(\lambda), \sum_{i=1, d}\left(\partial_{x^{i}} H^{i}(\lambda, x, t)-\eta^{\prime}(\lambda) \partial_{x^{i}} F^{i}(\lambda, x, t)\right)\right\rangle \varphi d x d t \\
& +\int_{\Omega \times \mathbb{R}_{+}}\left\langle\nu_{x, t}(\lambda), S(\lambda, x, t)\right\rangle \varphi d x d t+\int_{\Omega}\left\langle\nu_{x, t}(\lambda), \eta(\lambda)\right\rangle \varphi(x, 0) d x
\end{aligned}
$$

Moreover, the combination of the bounds (14), the approximation results (6) and (13) together with the available regularity of $\chi^{\kappa}, \varphi$ and the flux $H$ with respect to the space variable, implies

$$
\lim _{\Delta(h, \varepsilon) \longrightarrow 0} \sum_{i=2,7}\left|<\mu_{h, \varepsilon}^{i}, \varphi>_{\mathcal{Q}_{T}}\right|=\lim _{\Delta(h, \varepsilon) \longrightarrow 0} \sum_{m=1,4}\left|\mathcal{R}_{m}^{h, \varepsilon}\right|=0 .
$$

We recall that the main difficulty encountered in [4] lies in the evaluation of the dissipative term $\left\langle\mu_{h, \varepsilon}^{1}, \varphi>\right.$. We have then proved that

$$
\liminf _{\substack{\Delta(\varepsilon, h) \rightarrow 0 \\ \frac{\sqrt{\tau^{-}}}{\sqrt{\tau^{-}}} \rightarrow 0}}<\mu_{h, \varepsilon}^{1}, \varphi>\geq 0 .
$$


The proof of this last inequality combines similar arguments as those used in (87) together with the weak $B V$ estimate (iii) in (75), the inequality (67) and the following suitable decomposition (used in order to control the sign of the difference $\varphi_{k}^{n}-\varphi_{l}^{n}$ with quantities that go to zero as $\varepsilon \rightarrow 0$ )

$$
\varphi_{k}^{n}-\varphi_{l}^{n}=\underbrace{\left(\varphi_{k}^{n}-\varphi_{l}^{n}+A-B\right)}_{-C \varepsilon \leq \ldots . \leq 0}+\underbrace{(B-A)}_{0 \leq \ldots . \leq C \varepsilon}
$$

with

$$
A=\inf _{k \in K} \inf _{x \in B\left(x_{k}, C \varepsilon\right)}\left(\varphi(x, t)-\varphi\left(x_{k}, t\right)\right), \quad B=\sup _{k \in K} \sup _{x \in B\left(x_{k}, C \varepsilon\right)}\left(\varphi(x, t)-\varphi\left(x_{k}, t\right)\right) .
$$

By contrast, in the present convergence, we still have to deal with the evaluation of the volume approximation $\left(\mu_{\kappa^{\prime}, \varepsilon}^{0}, \varphi\right)_{h, T}$ which needs a more careful treatment. With this end in view, note that on account of the estimate $(65)(i)$ and the fact that $\operatorname{supp}(\theta(x)) \subset \partial \Omega \times\left[0, \kappa^{\prime}\right]$ together with the Lipschitz continuity of the numerical flux $\hbar$, we arrive at

$$
\left|\Gamma(\varphi)_{h, T}^{\kappa^{\prime}}\right| \leq C \operatorname{meas}(\partial \Omega)\left(\left\|\bar{u}_{h}\right\|_{\infty},\|b\|_{\infty}\right) \frac{\kappa^{\prime}}{\varepsilon} .
$$

This clearly shows the boundedness of this term according to the assumption $\kappa^{\prime}=$ $O(\varepsilon)$.

Let us now denote by

$$
\vartheta_{h, \varepsilon}^{\kappa^{\prime}}(x, t)=\theta(x) \hbar\left(\bar{n}(x), \bar{u}_{h}(x, t), b_{\kappa^{\prime}}(x, t)\right), \quad \bar{\varphi}=\varphi(x(\bar{x}, 0), t),
$$

thus, one may write

$$
\begin{aligned}
& \Gamma(\varphi)_{h, T}^{\kappa^{\prime}} \underbrace{=}_{\text {by }(81)} \sum_{\substack{\left\{n, t^{n} \leq T\right\} \\
k \in K}} \chi_{B_{k}} \chi_{\left[t^{n}, t^{n+1}[\right.}\left\langle\vartheta_{h, \varepsilon}^{\kappa^{\prime}}, \varphi\right\rangle+\mathcal{E}^{h, T}\left(\vartheta_{h, \varepsilon}^{\kappa^{\prime}} \varphi\right) \\
& =\int_{\Omega \times \mathbb{R}_{+}} \vartheta_{h, \varepsilon}^{\kappa^{\prime}}(x, t) \varphi d x d t+\mathcal{E}^{h, T}\left(\vartheta_{h, \varepsilon}^{\kappa^{\prime}} \varphi\right) \\
& =\int_{\Omega \times \mathbb{R}_{+}} \vartheta_{h, \varepsilon}^{\kappa^{\prime}}(x, t)(\bar{\varphi}+(\varphi-\bar{\varphi})) d x d t+\mathcal{E}^{h, T}\left(\vartheta_{h, \varepsilon}^{\kappa^{\prime}} \varphi\right) \\
& \underbrace{=}_{\text {by }(16)} \int_{\partial \Omega \times \mathbb{R}_{+}} \underbrace{\left(\int_{0}^{3 \kappa} \vartheta_{h, \varepsilon}^{\kappa^{\prime}}(x(\bar{x}, y), t) J(\bar{x}, y) d y\right)}_{\equiv \bar{\vartheta}_{h, \varepsilon}^{\kappa^{\prime}}(\bar{x}, t)} \bar{\varphi}(\bar{x}, t) d \bar{x} d t \\
& +\int_{\Omega \times \mathbb{R}_{+}} \vartheta_{h, \varepsilon}^{\kappa^{\prime}}(x, t)(\varphi-\bar{\varphi}) d x d t+\mathcal{E}^{h, T}\left(\vartheta_{h, \varepsilon}^{\kappa^{\prime}} \varphi\right) \\
& =\int_{\partial \Omega \times \mathbb{R}_{+}} \bar{\vartheta}_{h, \varepsilon}^{\kappa^{\prime}}(\bar{x}, t) \bar{\varphi}(\bar{x}, t) d \bar{x} d t \\
& +\int_{\Omega \times \mathbb{R}_{+}} \vartheta_{h, \varepsilon}^{\kappa^{\prime}}(x, t)(\varphi-\bar{\varphi}) d x d t+\mathcal{E}^{h, T}\left(\vartheta_{h, \varepsilon}^{\kappa^{\prime}} \varphi\right) .
\end{aligned}
$$

Denote by $\mathcal{R}\left(\vartheta_{h, \varepsilon}^{\kappa^{\prime}}, \varphi-\bar{\varphi}\right)$ the second integral term in the above inequality. Thus, the quadrature error (5) with similar arguments used in (89) implies

$$
\text { (i) }\left|\mathcal{E}^{h, T}\left(\vartheta_{h, \varepsilon}^{\kappa^{\prime}} \varphi\right)\right| \leq C \frac{h}{\varepsilon}, \quad(i i) \quad\left|\mathcal{R}\left(\vartheta_{h, \varepsilon}^{\kappa^{\prime}}, \varphi-\bar{\varphi}\right)\right| \leq C \kappa^{\prime} \times \frac{\kappa^{\prime}}{\varepsilon} .
$$


The assumption $h=o\left(\varepsilon^{2}\right)$ (in particular $h=o(\varepsilon)$ ), together with (91) (i) and (89) yield that the sequence $\vartheta_{h, \varepsilon}^{\kappa^{\prime}} \in L^{1}(\Omega \times[0, T])$. Moreover, on account of (91) (ii), the sequence $\bar{\vartheta}_{h, \varepsilon}^{\kappa^{\prime}}$ is bounded in $\in L^{1}(\partial \Omega \times[0, T])$. Thus, there exists a subsequence which still is denoted by $\bar{\vartheta}_{h, \varepsilon}^{\kappa^{\prime}}$ converging to some bounded Radon measure $\vartheta(\bar{x}, t)$ for the topology $\sigma\left(M_{b}, C_{c}\right)$ i.e.

$$
\int_{\partial \Omega \times \mathbb{R}_{+}} \bar{\vartheta}_{h, \varepsilon}^{\kappa^{\prime}}(\bar{x}, t) \bar{\varphi}(\bar{x}, t) d \bar{x} d t \underset{\substack{\Delta(h, \varepsilon) \rightarrow 0 \\ \kappa^{\prime} \longrightarrow 0}}{\longrightarrow}\langle\vartheta(\bar{x}, t), \bar{\varphi}\rangle, \quad \forall \bar{\varphi} \in C_{c}(\partial \Omega \times[0, T]) .
$$

Consequently,

$$
\Gamma(\varphi)_{h, T}^{\kappa^{\prime}} \underset{\substack{\Delta(h, \varepsilon)-0 \\ \kappa^{\prime} \longrightarrow 0}}{\longrightarrow}\langle\vartheta(\bar{x}, t), \bar{\varphi}\rangle, \quad \forall \bar{\varphi} \in C_{c}(\partial \Omega \times[0, T]) .
$$

In view of the results (86-88) and the above limit, the inequality (82) implies

$$
\mathcal{M}^{\eta}\left(\nu_{x, t}, \mathbf{a}, \varphi\right)-\langle\vartheta(\bar{x}, t), \bar{\varphi}\rangle \geq 0 .
$$

Therefore, the inequality (47) follows by taking $\varphi \in \mathcal{C}_{c}^{1}\left(\Omega \times \mathbb{R}_{+}\right)$.

Third step: Derivation of (53). Take $\left(\eta^{\delta}, H^{\delta}\right)$ defined in Remark (4.3) as an entropy-entropy flux pair $(\eta, H)$ in (82) and denote by $\hbar^{\delta}$ the numerical flux associated with the flux $H^{\delta}$, then one first applies the inequality (68) to get

$$
\hbar^{\delta}(n, u, v) \geq q_{r}^{\delta}(u, c, x, t) . n+\operatorname{sgn}_{\delta}(v-c)(g(n, u, v)-F(c, x, t) . n),
$$

with

$$
q_{r}^{\delta}(u, c, x, t)=\int_{c}^{u}\left(\operatorname{sgn}_{\delta}(w-c)-\operatorname{sgn}_{\delta}(u-c)\right) \partial_{w} F(w, x, t) d w .
$$

Secondly, as before, denoting by $\psi_{\delta}=\varphi \operatorname{sgn}_{\delta}(b-c)$ and

$$
\vartheta_{h, \varepsilon}^{\kappa^{\prime}}(x, t)=\theta(x) g\left(\bar{n}(x), \bar{u}_{h}(x, t), b_{\kappa^{\prime}}(x, t)\right), \quad \bar{\psi}_{\delta}=\psi_{\delta}(x(\bar{x}, 0), t),
$$

one may write

$$
\begin{array}{r}
\Gamma(\varphi)_{h, T}^{\kappa^{\prime}} \underbrace{\leq}_{\text {by }(94)} \underbrace{\Gamma(\varphi)_{h, T}^{\kappa^{\prime}, \delta}}_{\text {see below }}-\left(\operatorname{sgn}_{\delta}\left(b_{\kappa^{\prime}}-c\right) F(c, x, t) D_{\varepsilon, s}^{*} \chi^{\kappa}, \varphi\right)_{h, T} \\
\quad+\left(q_{r}^{\delta}\left(\bar{u}_{h}, c, x, t\right) D_{\varepsilon, s}^{*} \chi^{\kappa}, \varphi\right)_{h, T} \\
\underbrace{=}_{\text {by }(81)} \Gamma(\varphi)_{h, T}^{\kappa^{\prime}, \delta}-\int_{\Omega \times \mathbb{R}^{+}} F(c, x, t) \psi_{\delta} \nabla\left(1-\chi^{\kappa}\right) d x d t \\
+\int_{\Omega \times \mathbb{R}^{+}} q_{r}^{\delta}\left(\bar{u}_{h}, c, x, t\right) \varphi \nabla\left(1-\chi^{\kappa}\right) d x d t \\
+\mathcal{E}^{h, T}\left(F(c, x, t) \nabla\left(1-\chi^{\kappa}\right) \psi_{\delta}\right) \\
+\mathcal{E}^{h, T}\left(q_{r}^{\delta}\left(\bar{u}_{h}, c, x, t\right) \nabla\left(1-\chi^{\kappa}\right) \varphi\right),
\end{array}
$$


with

$$
\begin{gathered}
\Gamma(\varphi)_{h, T}^{\kappa^{\prime}, \delta} \quad:=\sum_{k \in K} \tau^{n} w_{k}^{n} \varphi_{k}^{n} \theta_{k}^{n} \operatorname{sgn}_{\delta}\left(b_{k}^{n}-c\right) g\left(\tilde{n}_{k}, u_{k}^{n}, b_{k}^{n}\right) \\
\underbrace{=}_{\text {as in }(90)} \int_{\partial \Omega \times \mathbb{R}^{+}} \bar{\vartheta}_{h, \varepsilon}^{\kappa^{\prime}}(\bar{x}, t) \bar{\psi}_{\delta} d \bar{x} d t+\mathcal{E}^{h, T}\left(\vartheta_{h, \varepsilon}^{\kappa^{\prime}} \psi_{\delta}\right) \\
+\mathcal{R}\left(\vartheta_{h, \varepsilon}^{\kappa^{\prime}}, \psi_{\delta}-\bar{\psi}_{\delta}\right) .
\end{gathered}
$$

So that, as in the previous step, one deduces that there exists a bounded Radon measure $\vartheta(\bar{x}, t)$ such that

$$
\Gamma(\varphi)_{h, T}^{\kappa^{\prime}, \delta} \underset{\substack{\Delta(h, s) \longrightarrow 0 \\ \kappa^{\prime} \longrightarrow 0}}{\longrightarrow}\left\langle\vartheta(\bar{x}, t), \operatorname{sgn}_{\delta}(b-c) \bar{\varphi}\right\rangle, \quad \forall \bar{\varphi} \in C_{c}(\partial \Omega \times[0, T]) .
$$

On the other hand, since we have in the weak star limit that

$$
\lim _{\Delta(\varepsilon, h) \rightarrow 0} q_{r}^{\delta}\left(\bar{u}_{h}, c, x, t\right)=\left\langle\nu_{x, t}, q_{r}^{\delta}(\lambda, c, x, t)\right\rangle,
$$

then, by using the following splitting

$$
q_{r}^{\delta}\left(\bar{u}_{h}, c, x, t\right)=\left\langle\nu_{x, t}, q_{r}^{\delta}(\lambda, c, x, t)\right\rangle+\left(q_{r}^{\delta}\left(\bar{u}_{h}, c, x, t\right)-\left\langle\nu_{x, t}, q_{r}^{\delta}(\lambda, c, x, t)\right\rangle\right),
$$

together with the fact that $\kappa^{\prime}=O(\kappa)$ and Szepessy's weak trace limit (Lemma 1.1 in [41]), one gets

$$
\lim _{\substack{(\varepsilon, h) \rightarrow 0 \\ \kappa^{\prime} \rightarrow 0}} \int_{\Omega \times \mathbb{R}^{+}} q_{r}^{\delta}\left(\bar{u}_{h}\right) \varphi \nabla\left(1-\chi^{\kappa}\right) d x d t=\int_{\partial \Omega \times \mathbb{R}^{+}}\left\langle\gamma \nu_{x, t}, q_{r}^{\delta}(\lambda)\right\rangle \varphi d \sigma(x) d t .
$$

We also have

$$
\lim _{\kappa^{\prime} \rightarrow 0} \int_{\Omega \times \mathbb{R}^{+}} F(c, x, t) \psi_{\delta} \nabla\left(1-\chi^{\kappa}\right) d x d t=\int_{\partial \Omega \times \mathbb{R}^{+}} \operatorname{sgn}_{\delta}(b-c) F(c, x, t) \varphi d \sigma(x) d t .
$$

In view of the results (96-98), the inequality (95) yields

$$
\begin{aligned}
\lim _{\substack{\Delta(\varepsilon, h) \rightarrow 0 \\
\kappa^{\prime} \rightarrow 0}}\left(\Gamma(\varphi)_{h, T}^{\kappa^{\prime}} \leq\right. & \int_{\partial \Omega \times \mathbb{R}^{+}} \\
& \operatorname{sgn}_{\delta}(b-c) \varphi d \vartheta(\sigma(x), t) \\
& -\int_{\partial \Omega \times \mathbb{R}^{+}} \operatorname{sgn}_{\delta}(b-c) F(c, x, t) \varphi d \sigma(x) d t \\
& +\int_{\partial \Omega \times \mathbb{R}^{+}}\left\langle\gamma \nu_{x, t}, q_{r}^{\delta}(\lambda, c, x, t)\right\rangle \varphi d \sigma(x) d t .
\end{aligned}
$$

On account of the results (86-88) with $\eta=\eta^{\delta}$ and the bound (51), the inequality (82) gives

$$
\begin{aligned}
\mathcal{M}^{\eta_{c}^{\delta}}\left(\nu_{x, t}, \mathbf{a}, \varphi\right) & -\int_{\partial \Omega \times} \operatorname{sgn}_{\delta}(b-c) \varphi d \vartheta(\sigma(x), t) \\
& +\int_{\partial \Omega \times} \operatorname{sgn}_{\delta}(b-c) F(c, x, t) \varphi d \sigma(x) d t \geq C \delta\|\varphi\|_{\infty}
\end{aligned}
$$

By letting $\delta \rightarrow 0$ and using Lebesgue's theorem, the proof of (53) is completed. 
Appendix A. Denoting by $F_{i}=F\left(b\left(x_{i}, t\right), x_{i}, t\right)$ (with $b$ a smooth function on $\bar{\Omega})$, one has to evaluate the term

$$
\mathcal{K}^{h, \varepsilon}=\sum_{(k, l) \in(K \times G)} w_{k} \varphi_{k} \tilde{w}_{l}\left(F_{k}+F_{l}\right) \mathcal{A}_{k l} .
$$

To this end, let us introduce the characteristic function $\chi$ of the domain $\Omega$ and use the notation $\chi_{i}=\chi\left(x_{i}\right)$; then, one may write, on the one hand,

$$
\begin{aligned}
& \sum_{(k, l) \in(K \times G)} w_{k} \varphi_{k} \tilde{w}_{l} F_{l} \mathcal{A}_{k l}=\sum_{(k, l) \in(K \cup G)^{2}} \chi_{k}\left(1-\chi_{l}\right) \tilde{w}_{k} \tilde{w}_{l} \varphi_{k} F_{l} \mathcal{A}_{k l} \\
&= \sum_{k \in K} \chi_{k} w_{k} \varphi_{k}\left(\tilde{D}_{\varepsilon, s} F\right)_{x=x_{k}}-\sum_{(k, l) \in(K \times K)} w_{k} w_{l} \chi_{k} \chi_{l} \varphi_{k} F_{l} \mathcal{A}_{k l} \\
& \underbrace{\approx}_{(11),(6)} \int_{\Omega} \varphi \operatorname{div} F(b(x, t), x, t) d x-\sum_{(k, l) \in(K \times K)} w_{k} w_{l} \chi_{k} \chi_{l} \varphi_{k} F_{l} \mathcal{A}_{k l} \\
&=\int_{\Omega} \varphi \operatorname{div} F(b(x, t), x, t) d x+I
\end{aligned}
$$

on the other hand,

$$
\begin{aligned}
& \sum_{(k, l) \in(K \times G)} w_{k} \varphi_{k} \tilde{w}_{l} F_{k} \mathcal{A}_{k l} \\
&= \sum_{(k, l) \in(K \times G)} w_{k} \tilde{w}_{l} \varphi_{l} F_{k} \mathcal{A}_{k l}+\sum_{(k, l) \in(K \times G)} w_{k} \tilde{w}_{l}\left(\varphi_{k}-\varphi_{l}\right) F_{k} \mathcal{A}_{k l} \\
&= \sum_{(k, l) \in(K \cup G)^{2}} \chi_{k}\left(1-\chi_{l}\right) \tilde{w}_{k} \tilde{w}_{l} \varphi_{l} F_{k} \mathcal{A}_{k l}+\sum_{(k, l) \in(K \times G)} w_{k} \tilde{w}_{l}\left(\varphi_{k}-\varphi_{l}\right) F_{k} \mathcal{A}_{k l} \\
& \underbrace{\approx}_{(11),(6)} \int_{\Omega} F(b(x, t), x, t) \nabla \varphi d x-\sum_{(k, l) \in(K \times K)} w_{k} w_{l} \chi_{k} \chi_{l} \varphi_{l} F_{k} \mathcal{A}_{k l} \\
& \quad+\sum_{(k, l) \in(K \times G)} w_{k} \tilde{w}_{l}\left(\varphi_{k}-\varphi_{l}\right) F_{k} \mathcal{A}_{k l} \\
&=\int_{\Omega} F(b(x, t), x, t) \nabla \varphi d x+I^{\prime}+I I .
\end{aligned}
$$

Therefore, one combines the two results to get

$$
\mathcal{K}^{h, \varepsilon} \approx \int_{\partial \Omega} F(b(x, t), x, t) \cdot n \varphi(x) d \sigma(x)+I^{\prime}+I+I I .
$$

By switching the indices $k$ and $l$ and using that $\mathcal{A}_{k l}=-\mathcal{A}_{l k}$, one gets that $I^{\prime}+I=0$. Moreover, the estimates (14) imply that $|I I| \leq C \operatorname{meas}(\partial \Omega) \kappa^{\prime}$. Consequently,

$$
\mathcal{K}^{h, \varepsilon} \underset{\substack{\Delta(h, \varepsilon)-\\ \kappa^{\prime} \longrightarrow 0}}{\longrightarrow} \int_{\partial \Omega} F(b(x, t), x, t) . n \varphi(x) d \sigma(x)
$$

and the proof is completed. 
Appendix B. To prove the equivalence between definitions (4.2) and (4.5), it is sufficient to show that (4.5) implies (4.2), since the converse is obvious. To this end, the main difficulty lies in establishing that the Radon measure $\vartheta_{s, t}$ is related to the Young measure $\gamma \nu_{s, t}$ in the following sense

$$
d \vartheta_{s, t}=\left\langle\gamma \nu_{s, t}, F(\lambda, s, t)\right\rangle d \sigma(s) d t .
$$

Indeed, using the notation $\mathcal{M}^{\eta}\left(\nu_{x, t}, \varphi\right)$ in (52), the inequality (47) to be proved reads

$$
\mathcal{M}^{\eta}\left(\nu_{x, t}, \varphi\right)-\int_{\partial \Omega \times \mathbb{R}_{+}}\left\langle\gamma \nu_{s, t}, B(\lambda, s, t)\right\rangle \cdot n(s) \varphi(s, t) d s d t \geq 0 .
$$

Taking the following decomposition

$$
\varphi(x, t)=\varphi(x, t) \chi_{\kappa}(x(\bar{x}, y))+\varphi(x, t)\left(1-\chi_{\kappa}(x(\bar{x}, y))\right),
$$

where $\chi_{\kappa}$ is defined by (25), the inequality (102) becomes

$$
\begin{array}{r}
\mathcal{M}^{\eta}\left(\nu_{x, t}, \varphi(x, t)\left(\chi_{\kappa}(x(\bar{x}, y))\right)+\mathcal{M}^{\eta}\left(\nu_{x, t}, \varphi(x, t)\left(1-\chi_{\kappa}(x(\bar{x}, y))\right)\right)\right. \\
-\int_{\partial \Omega \times \mathbb{R}_{+}}\left\langle\gamma \nu_{s, t}, B(\lambda, s, t)\right\rangle . n(s) \varphi(s, t) d s d t \geq 0 .
\end{array}
$$

Since $\varphi \chi_{\delta} \in \mathcal{C}_{c}^{1}\left(\Omega \times \mathbb{R}_{+}\right)$, then, the term $\mathcal{M}^{\eta}\left(\nu_{x, t}, \varphi(x, t)\left(\chi_{\kappa}(x(\bar{x}, y))\right)\right.$ is positive thanks to (52). Thereby, to prove (102), it suffices to show that

$$
\begin{aligned}
\mathcal{M}^{\eta}\left(\nu_{x, t}, \varphi(x, t)(1\right. & \left.\left.-\chi_{\kappa}(x(\bar{x}, y))\right)\right) \\
& -\int_{\partial \Omega \times \mathbb{R}_{+}}\left\langle\gamma \nu_{s, t}, B(\lambda, s, t)\right\rangle \cdot n(s) \varphi(s, t) d s d t \geq 0 .
\end{aligned}
$$

By developing the derivative $\nabla\left(\varphi\left(1-\chi_{\kappa}\right)\right)$, a straightforward calculation proves that

$$
\lim _{\kappa \rightarrow 0^{+}} \mathcal{M}^{\eta}\left(\nu_{x, t}, \varphi\left(1-\chi_{\kappa}\right)\right)=\int_{\partial \Omega \times \mathbb{R}_{+}}\langle\gamma \nu, \hbar(\lambda, x, t)\rangle \cdot n \varphi d \sigma(x) d t .
$$

Therefore, by tending $\kappa \rightarrow 0^{+}$in the previous inequality, we get

$$
\begin{aligned}
\int_{\partial \Omega \times \mathbb{R}_{+}}\left\langle\gamma \nu_{x, t}, \hbar(\lambda, x, t)\right\rangle \cdot n(x) d \sigma(x) d t & \\
& -\int_{\partial \Omega \times \mathbb{R}_{+}}\left\langle\gamma \nu_{s, t}, B(\lambda, s, t)\right\rangle \cdot n(s) \varphi(s, t) d s d t \geq 0 .
\end{aligned}
$$

To prove this inequality, one first starts with (53) and introduces the decomposition (103). Secondly, by letting $\kappa \rightarrow 0^{+}$and using the same arguments as before, one obtains

$$
\begin{gathered}
\int_{\partial \Omega \times \mathbb{R}^{+}}(\langle\gamma \nu, q(\lambda, c, x, t) \cdot n\rangle+\operatorname{sgn}(b-c) F(c, x, t) \cdot n) \varphi d \sigma(x) d t \\
-\int_{\partial \Omega \times \mathbb{R}^{+}} \operatorname{sgn}(b-c) \varphi d \vartheta(\sigma(x), t) \geq 0 .
\end{gathered}
$$


As in [42], taking consecutively, $\left.c=1+\max \left\{\left\|u_{0}\right\|_{\infty},\|b\|_{\infty}\right)\right\}$ and $c=-1-$ $\left.\max \left\{\left\|u_{0}\right\|_{\infty},\|b\|_{\infty}\right)\right\}$, we find that

$$
\int_{\Gamma}\left\langle\gamma \nu_{x, t}, F(\lambda, x, t)\right\rangle \cdot n \varphi d \sigma(x) d t=\int_{\Gamma} \varphi d \vartheta(\sigma(x), t)
$$

which yields (101). By plugging this last identity into the inequality (105), one gets

$$
\begin{aligned}
\int_{\partial \Omega \times \mathbb{R}^{+}} & \langle\gamma \nu, q(\lambda, c, x, t)\rangle \cdot n \varphi d \sigma(x) d t \\
& -\int_{\partial \Omega \times \mathbb{R}^{+}}\langle\gamma \nu, \operatorname{sgn}(b-c)(F(\lambda, x, t)-F(c, x, t))\rangle \varphi d \sigma(x) d t \geq 0 .
\end{aligned}
$$

To obtain (104), it suffices to use the decomposition

$$
\operatorname{sgn}(b-c)(F(\lambda, x, t)-F(c, x, t))=\operatorname{sgn}(b-c)(F(\lambda, x, t)-F(b, x, t))-q(b, c, x, t)
$$

and to approximate any entropy function $\eta \in \mathcal{C}^{1}$ by the following functions $\eta_{n}(s)=$ $\sum_{i=1}^{n} \alpha_{i}^{(n)}\left|s-k_{i}^{(n)}\right|$

To end the proof of $(4.5) \Longrightarrow(4.2)$, one needs to show that (52) implies the initial condition (48). This is achieved by Theorem 2.2 in [2].

Acknowledgement. The author is grateful to two referees for valuable comments and suggestions. This work has been partly supported by the European network HYKE, funded by the EC contract HPRN-CT-2002-00282.

\section{REFERENCES}

[1] Bardos C., Leroux A.Y., Nedelec J.C., First order quasilinear equations with boundary conditions, Com. Part. Diff. Equ., 4, 9 (1979), pp. 1017-1034.

[2] Benharbit S., Chalabi A., Vila J.P., Numerical viscosity, and convergence of finite volume Methods for conservation laws with boundary conditions, SIAM J. Numer. Anal., 32:3 (1995), pp. 775-796.

[3] Ben Moussa B., Szepessy A., Scalar Conservation Laws with Boundary Conditions and Rough Data Measure Solutions, Methods and Applications of Analysis, 9:4 (2002), pp. 579-598.

[4] Ben Moussa B., Vila J.P., Convergence of SPH method for scalar nonlinear conservation laws, SIAM J. Numer. Anal., 37:3 (2000), pp. 863-887.

[5] Ben Moussa B., N. Lanson., Vila J.P., Convergence of Meshless Methods for Conservation Laws Application to Euler equations, International Series of Nume maths. vol. 129, Verlag Basel/ Switzerland, (1999), pp. 97-108.

[6] Ben Moussa B., Analyse numérique de méthodes particulaires de type SPH pour les lois de conservation, PHD thesis INSA-Toulouse, January 20, 1998.

[7] Ben Moussa B., Lacome J.L., On renormalized formulation of SPH Method: Overview and improvements, in revised form.

[8] Ben Moussa B., Lacome J.L., SPH Method and Boundary Conditions Applications to Euler Equations, in preparation.

[9] Benz W., Smooth Particle Hydrodynamics : a Review, Harvard-Smithsonian Center for Astrophysics preprint 2884, (1989).

[10] Benz W., Asphaug A., Impact Simulations with Fracture, I. Methods and Tests, Icarus, 107 (1993), pp. 98-116.

[11] Cockburn B., Coquel F., LeFloch P., Convergence of finite volume method for multidimensional conservation laws, SIAM J. Numer. Anal., 32:3 (1995), pp. 687-705.

[12] Crandall M., Majda A., Monotone Difference Approximations for Scalar Conservation Laws, Math. of Comp., 34, 149 (1980), pp. 1-21. 
[13] Diperna R.J., Measure-valued solution to conservation laws, Arch. Rat. Mech. Anal., 88 (1985), pp. 223-270.

[14] Dubois F., Lefloch P., Boundary conditions for nonlinear hyperbolic systems of conservation laws, J.D.E., 71, 1 (1988), pp. 93-122.

[15] LuCY L., A numerical approach to the testing of the fission hypothesis, Astrono. J., 82 (1977), p. 1013.

[16] Harten A., Lax P.D., Van Leer B., On upstream differencing and Godunov type schemes for hyperbolic conservation laws, SIAM Rev., 25 (1983), pp. 35-61.

[17] Harten A., Hyman J.M., Self adjusting grid methods for one-dimensional hyperbolic conservation laws, J. Comp. Phys., 50 (1983), pp. 235-269.

[18] Herant, Dirty Tricks for SPH, Mem. S. A. It., XX (1993), pp. 1-10.

[19] Gingold R.A., Monaghan J.J., Shock simulation by the particle method SPH., J.C.P., 52 (1983), pp. 374-389.

[20] Gingold R.A., Monahghan, MNRAS, (1977), pp. 181-375.

[21] Johnson G.R., Beissel S.R., Normalized Smoothing functions for impact computations, Int. Jour. Num. Methods Eng., (1996).

[22] Kröner D., Rokyta M., Convergence of upwind finite volume schemes for scalar conservation laws in 2D, SIAM J. Numer. Anal, 31 (1994), pp. 324-343.

[23] Kruzkov S.N., First order quasilinear equations in several independent variables, Math. USSR Sbornik, 10 (1970), pp. 217-243.

[24] Kuznetsov N.N., Accuracy of some approximate methods for computing the weak solution of a first order quasi-linear equation, USSR Comp.Math. and Math. Phys., 16 (1976), pp. 105-119.

[25] Kuznetsov N.N., Volosin S.A., On monotone difference approximations for a first order quasilinear equation, Soviet Math. Dokl., 17 (1976), pp. 1203-1206.

[26] Lacome J.L., Analyse de la méthode particulaire SPH, Application à la Détonique, PHD thesis INSA-Toulouse, January 19, 1998.

[27] Lattanzio J.C., Monaghan J.J., Pongracic H., Schwarz M.P., Controlling penetration, SIAM J. Sci. Stat. Comput., 7, 2, 1986.

[28] Lax P.D., Shock waves and entropy, Contributions to non linear Functional analysis, ed. E.A. Zarantonello, Academic press, (1971), pp. 603-634.

[29] LuCy L., Numerical approach to testing the fission hypothesis, Astrono. J., 82 (1977), pp. 10131024.

[30] Lui G.R., Lui M.B., Smoothed Particle Hydrodynamics, World Scientific Publishing Co. Pte. Ltd. (2003)

[31] Malek J., Necas J., Rokyta M., Ruzicka M., Weak and Measure-valued Solutions to Evolutionary PDEs, Applied Mathematics and Mathematical Computation, ed. Chapman and Hall, 2, 1996.

[32] Mas-Gallic S., Raviart P.A., A Particle Method for First-order Symmetric Systems, Numer. Math., 51 (1987), pp. 323-352.

[33] Monaghan J.J., Simulating Gravity Currents with SPH. III : Boundary Forces., Report, 28 March, Monash university, (1995).

[34] Monaghan, Smooth Particle Hydrodynamics, Annu. Rev. Astron. Astrop., 30 (1992), pp. 543574.

[35] NoH W.F., Errors for calculations of strong shocks using an artificial viscosity and an artificial heat flux, JCP, 72 (1978), pp. 78-120.

[36] OSher S., Riemann solvers, the entropy condition and difference approximations, Siam Jour. Num. Anal, 21:2 (1984), pp. 217-235.

[37] Отто F., Initial-boundary value problem for scalar conservation law, C. R. Acad. Sci. Paris, t. 322, Serie I, (1996), pp. 729-734.

[38] Randles R.W., Libertsky L.D., Smoothed Particle Hydrodynamics, Some recent improvements and Applications, Comp. Meth. Appli. Mech. Eng., 138 (1996), pp. 375-408.

[39] Raviart P.A., An analysis of particle methods, in Num. method in fluid dynamics, F. Brezzi ed. Lecture Notes in Math., vol 1127, Berlin, Springer 1985.

[40] Roe P.L., Approximate Riemann solvers, parameter vectors and difference scheme, JCP, 43 (1981), pp. 357-372.

[41] Szepessy A., Measure Valued Solutions to Scalar Conservation Laws with Boundary conditions, Arch. Rat. Mech. Anal., (1989), pp. 181-193.

[42] SZEPESSY A., Convergence of a streamline diffusion finite element method for a conservation law with boundary conditions, RAIRO Model. Math. Anal. Numer., 25 (1991), pp. 749-783.

[43] TARTAR L., Compensated compactness and applications to partial differential equations, in Reaserach notes in Mathematics, nonlinear analysis, and mechanis: Heriot-Watt Sympo- 
sium, Vol 4, KNOPS, R.J,(ed.) New York: Ptman Press, 1979.

[44] Tadmor E., Numerical viscosity and the entropy condition, Math. of Comp., 43 (1984), pp. 369381.

[45] Vila J.P., On Particle weighthed methods and Smooth Particle Hydrodynamics, M3AS, 9:3 (1999), pp. 161-209.

[46] Zumbrun K., On a nonlocal dispersive equation modeling particle suspensions, Quarter. Appl. Math., LVII:3 (1999), pp. 573-600. 
B. BEN MOUSSA 